\title{
Industry 4.0 Applications for Medical/Healthcare Services
}

\author{
Shuva Paul ${ }^{1, *(1)}$, Muhtasim Riffat ${ }^{2}\left(\mathbb{D}\right.$, Abrar Yasir $^{2}$, Mir Nusrat Mahim $^{2}$, Bushra Yasmin Sharnali ${ }^{2}$, \\ Intisar Tahmid Naheen ${ }^{2}$, Akhlaqur Rahman ${ }^{3}$ (D) and Ambarish Kulkarni ${ }^{4}$ \\ 1 Georgia Institute of Technology, Atlanta, GA 30332, USA \\ 2 Department of Electrical Engineering and Computer Science, North South University, \\ Dhaka 1229, Bangladesh; muhtasim.riffat@northsouth.edu (M.R.); abrar.yasir@northsouth.edu (A.Y.); \\ nusrat.mahim@northsouth.edu (M.N.M.); bushra.yasmin@northsouth.edu (B.Y.S.); \\ intisar.naheen@northsouth.edu (I.T.N.) \\ 3 School of Industrial Automation, Engineering Institute of Technology, Melbourne, VIC 3000, Australia; \\ akhlaqur.rahman@eit.edu.au \\ 4 School of Engineering, Swinburne University of Technology, Hawthorn, Melbourne, VIC 3122, Australia; \\ ambarishkulkarni@swin.edu.au \\ * Correspondence: shuva.paul@jacks.sdstate.edu
}

Citation: Paul, S.; Riffat, M.; Yasir, A.; Mahim, M.N.; Sharnali, B.Y.; Naheen,

I.T.; Rahman, A.; Kulkarni, A.

Industry 4.0 Applications for Medical/Healthcare Services. J. Sens. Actuator Netw. 2021, 10, 43.

https: / / doi.org/10.3390/jsan10030043

Academic Editors: Igor Bisio and Claudio Savaglio

Received: 20 March 2021

Accepted: 24 June 2021

Published: 30 June 2021

Publisher's Note: MDPI stays neutral with regard to jurisdictional claims in published maps and institutional affiliations.

\begin{abstract}
At present, the whole world is transitioning to the fourth industrial revolution, or Industry 4.0, representing the transition to digital, fully automated environments, and cyber-physical systems. Industry 4.0 comprises many different technologies and innovations, which are being implemented in many different sectors. In this review, we focus on the healthcare or medical domain, where healthcare is being revolutionized. The whole ecosystem is moving towards Healthcare 4.0, through the application of Industry 4.0 methodologies. Many technical and innovative approaches have had an impact on moving the sector towards the 4.0 paradigm. We focus on such technologies, including Internet of Things, Big Data Analytics, blockchain, Cloud Computing, and Artificial Intelligence, implemented in Healthcare 4.0. In this review, we analyze and identify how their applications function, the currently available state-of-the-art technologies, solutions to current challenges, and innovative start-ups that have impacted healthcare, with regards to the Industry 4.0 paradigm.
\end{abstract}

Keywords: cloud computing method; artificial intelligence; IoT; blockchain; healthcare 4.0; industry 4.0

\section{Introduction}

Science and technology have been advancing in many ways, impacting almost every sector of human life. Advanced electrical power generation, transmission, and distribution systems (i.e., smart grids), as well as advanced healthcare, education, roading, and transportation, and so on, are making daily life more efficient and easy [1]. The use of Machine Learning (ML) in different sectors has helped to operate multiple interconnected industries in parallel [2-6]. Industry 4.0, or the fourth industrial revolution, describes the conversion and development of industrial manufacturing through the digitization of new technologies. The idea of the industrial revolution has varied from decade to decade; presently, there exists global competition between industrial manufacturers. In response, Industry 4.0 has added a new dimension where, with the help of new technologies, the industrial market has hit a new level, the likes of which has not been seen before. The industrial internet concept was introduced in 2012 [7], with a close-fitting mixture of both digital and physical worlds adjoining Big Data with the Internet of Things (IoT). This thought describes many widespread ideas about Industry 4.0. Experts have estimated that around $46 \%$ [8] of the global economy could benefit from the industrial internet. The industrial revolution can be presented or divided into four revolutions. The first industrial revolution, known as Industry 1.0, began with mechanical efficiency in the late 1800s [9] and involved the manual use of mechanical tools, mostly in the textile industry. The second revolution's 
central concept was electrification, which enabled mass production. The third revolution was characterized by digitization through the use of microelectronics and automation, such that flexible production became possible. Programmable machines, such as Network Computers (NCs) and Programmable Logic Controllers (PLCs), have been used to manufacture many products. The fourth, and current, revolution is the most remarkable one, introducing cyber-physical systems which consist of useful technological methods, such as IoT, Big Data Analytics (BDA), Cloud Computing, blockchain, and Artificial Intelligence (AI). The fourth revolution was triggered by the development of IT. The origins of the first concepts of Industry 4.0 are based on four topics, according to German manufacturers [10]:

- $\quad$ The benefits and appearance of IoT;

- The blending of technical methods;

- The virtualization of the real world; and

- The smart factory, where the word "smart" indicates Industrial Production (IP).

Industry 4.0 has become a topic of interest in recent years. Therefore, it has gained a lot of attention, and there has been much interest in the literature regarding Industry 4.0 from academics and practitioners alike.

\subsection{Literature Relevant to Healthcare Applications in Industry 4.0}

In this section, we review some of the literature that has focused on Industry 4.0 and its potential in medical and healthcare services under the 4.0 paradigm. A systematic survey of the e-Health ecosystem, along with the impacts on it from the IoT, Big Data technology, and Fog and Cloud Computing perspectives, has been presented in [11]. The authors discussed Industry 4.0 technology and its composition in the health sector. They described the primary technologies, their benefits, cross-disciplinary challenges, and the lessons learned from them. According to the authors of [12], there is currently a lack of systematic reviews regarding the state-of-the-art in the fourth industrial revolution. The authors aimed to address this gap by investigating academic progress in the Industry 4.0 paradigm and summarized current research activities, indicating deficiencies and proposing potential research directions. The authors of [13] proposed a sustainable framework for Industry 4.0. They provided a methodical literature review of 85 papers focused on Industry 4.0 classified into five research categories: IoT, BDA, Cloud Computing, blockchain, and AI. The authors argued that, even with the attention that Industry 4.0 has gotten, there are still minimal systematic and extensive reviews showcasing this paradigm's dynamic nature. They listed the various research pathways used in Industry 4.0 and discussed the current state of research in the domains of Industry 4.0. Another study [14] mainly focused on Healthcare 4.0 and how it is derived from Industry 4.0. Noting the broad spectrum of technologies enabling Industry 4.0 and Healthcare 4.0 to provide more efficient and effective services, the authors used the Wisdom Pyramid Methodology. They conducted a systematic review of presently available digital technologies at the forefront of Healthcare 4.0. The authors of [15] explored the existing gaps, challenges, and tendencies in implementing Healthcare 4.0. They found that Healthcare 4.0 research has been conducted in an interdisciplinary manner, with diverse applications and functions and discovered Healthcare 4.0 implementations in hospital information management. The authors provided practical orientation and academic alignment with their study. Table 1 provides a summary of some of the existing literature related to Healthcare 4.0 and Industry 4.0. 
Table 1. Existing literature on Industry 4.0 and Healthcare 4.0.

\begin{tabular}{lll}
\hline References & Technology & Methodology \\
\hline$[16]$ & IoT and BDA & Defragmenting brain signals \\
{$[17]$} & IoT and BDA & Health-sensing devices \\
{$[18]$} & IoT and BDA, Cloud Computing & Generation of data for neuroscience applications \\
\hline$[19]$ & blockchain & Countering counterfeiting of drugs \\
{$[16,20-27]$} & blockchain & Distributed attribute-based signature scheme for healthcare records \\
{$[28-37]$} & blockchain, Cloud Computing & Decentralized healthcare record management system and healthcare data-sharing services \\
{$[38-40]$} & blockchain & Healthcare data gateway and data access control policy for EHRs \\
{$[41]$} & blockchain & Platform for safe sharing of genomic data \\
{$[42]$} & blockchain & Mobile-based healthcare record sharing system \\
{$[43,44]$} & blockchain & Personalized medicine system \\
{$[45]$} & blockchain, IoT & Increased accountability in supply chains \\
{$[46-48]$} & blockchain & Performance evaluation on blockchain frameworks and hyperledger fabric framework \\
{$[49,50]$} & blockchain & Redesign of blockchain systems to increase throughput and reduction of time in sharing EHRs \\
{$[51]$} & blockchain & while enabling widespread implementation of blockchain EHR systems \\
{$[52]$} & blockchain & Remote continuous patient monitoring system \\
\hline$[53]$ & AI & Privacy-preserving predictive healthcare modeling framework \\
{$[54]$} & AI & Observing virus-affected patient activities to lower spread \\
{$[55]$} & AI & Automatic detection and removal of misinformation related to viruses on social media \\
\hline$[56]$ & Cloud Computing & Optimization of clinical trials for drugs and vaccines using robots to lower risks \\
{$[57]$} & Cloud Computing & Delivery model for healthcare sector \\
{$[58]$} & Cloud Computing & Scalable infrastructure, security models, and fast access to information \\
{$[59]$} & Cloud Computing & Cloud infrastructure as a service \\
\hline
\end{tabular}

\subsection{Contribution}

We focused on four key technologies that have a general impact on Healthcare 4.0 and Industry 4.0; that is, we analyzed the present state of IoT, BDA, blockchain, AI, and Cloud Computing in Healthcare 4.0, within the sphere of the Industry 4.0 paradigm. We put significant focus on blockchain technology, while also analyzing and discussing applications and implementations of AI, Cloud Computing, IoT, and blockchain in Healthcare 4.0 scenarios. We discuss modern deployments of these technologies in Healthcare 4.0, determined through our thorough literature review. We also analyzed state-of-the-art applications which are either in use or in the prototype stage. The applications and methods used for the state-of-the-art applications are compiled in Table 1. Another subject that was not found in any of the comparable review papers was start-ups related to these technologies in the Healthcare 4.0 and Industry 4.0 scenarios; therefore, we analyze and discuss innovative technologies and start-ups related to this industry and their impact. This part is compiled in Table 2, which can be used to gather a brief overview. Finally, we also discuss the challenges and research gaps faced while trying to progress in this sector using new Industry 4.0 technologies.

\subsection{Organization}

The rest of the paper is organized as follows: Section 2 discusses the industrial revolutions in detail, its definition, architecture, and so on. Applications of Industry 4.0 in medical/healthcare services are discussed, along with state-of-the-art research works applying IoT, BDA, blockchain, and AI, in Section 3. Section 4 discusses the research gaps and potential research directions along this line. Finally, this article concludes with a summary of the contributions of this article in Section 5.

\section{Industrial Revolutions}

The industrial revolution has been defined as the alteration towards novelty of procedure in some places in Europe and the United States, from 1760 up to the end of 1840 [60]. The world started to change how manufacturing, production, and transportation was conducted, compared to the older methods, through the use of newer technology and more efficient methodologies. The period mentioned above is now called the first industrial rev- 
olution, which was the first of its kind to usher in a new age of progress and advancements. With the fourth industrial revolution now upon us, we can divide the past revolutions into three distinct periods. Figure 1 presents the timeline of industrial revolutions and their features.

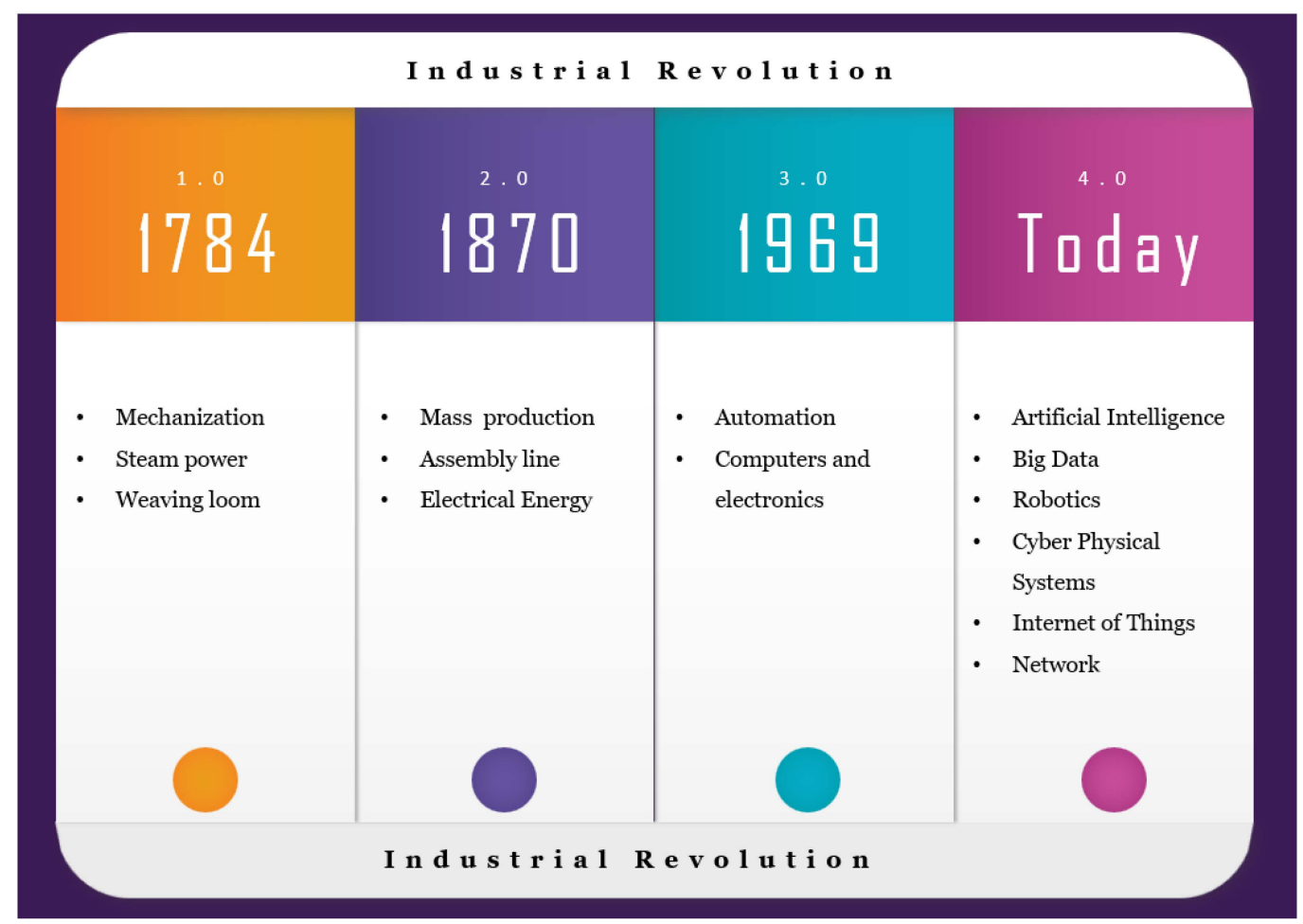

Figure 1. Industrial revolutions (from Industry 1.0 to Industry 4.0) and their features.

The first industrial revolution was arguably the most influential and essential transition period in human history, starting our stride towards the "modern age" from its inception. Notable developments include textile machinery, steam power, machine tools, telegraph communication, electric generators, modern factories, and improved canals, roads, and railways. Before this revolution, the world was still dependent on agriculture; however, with the advent of mechanization, the world transitioned from agriculture to industry as the backbone of the social economy.

The second industrial revolution happened about a century after the first revolution. It started in the late 19th century and continued until the early 20th century, roughly between 1870 and 1914, before World War I [61]. It is also known as the Technological Revolution, and was characterized by rapid standardization and industrialization. A notable event in this revolution was the rise of new energy sources, mainly electricity, oil, and gas. The internal combustion engine was one of the most important inventions during this period. It allowed for faster and more convenient transportation to be invented, such as automobiles and airplanes. Communication also advanced tremendously, with the invention of telegraphs and telephones. In terms of innovations, many have argued that the second revolution, also known as Industry 2.0, has been the most impactful one.

For the third industrial revolution, we again had to wait another 100 years. The rise of new technologies and energy sources brought upon the digital revolution, occurring in the middle of the 20th century. Electronics, computers, and telecommunications came into the picture, which enabled space expeditions, experimental research, and biotechnology. Another major event from this time is the high level of automation that became available thanks to the invention of programmable robots and controllers. Nuclear energy was another vital invention that was a part of the third industrial revolution [62]. 


\subsection{Definition of Industry 4.0}

The digital transformation of consecutive manufacturing and artificial practices through modern smart technologies basically describes Industry 4.0. The basis of this revolution is cyber-physical systems, or intelligent machines [63]. The integration of IoT, increased automation technology, and improved communication and self-counseling methods are the main characteristics of Industry 4.0 [64]. Schwab [65] has predicted that this revolution will see emerging technologies in AI, robotics, nanotechnology, biotechnology, IoT, decentralized systems, 3D printing, fifth-generation communication technologies, quantum computing, and autonomous vehicles. Industry 4.0 arose in the early 2010s in Germany, where the German government promoted manufacturing computerization under its high-tech strategy project. The Industry 4.0 principle has been applied to many different aspects and re-branded as necessary by other sectors or different companies. For example, under M4 (Meggitt, Modular, Modifiable, and Manufacturing) [66], an Aerospace manufacturer has branded its Industry 4.0 projection. The digitization phase in Industry 4.0 and the effect it is expected to have on the labor market has been observed under the topic work 4.0 [67]. Different terms may be used for other sectors, but all of these follow the principles of Industry 4.0 and fall under the umbrella of Industry 4.0. Therefore, they are all part of the same revolution.

\subsection{Architecture of Industry 4.0}

Industry 4.0 is a term that describes many different sections of a whole, which all work in different ways in their bubble to enable the next revolution. Even though other parts of this whole concept work differently, they all share common goals and principles. These principles and objectives can be thought of as the architecture of Industry 4.0. Four design principles have been identified as integral for Industry 4.0, inspired by [68], which are shown in Figure 2.

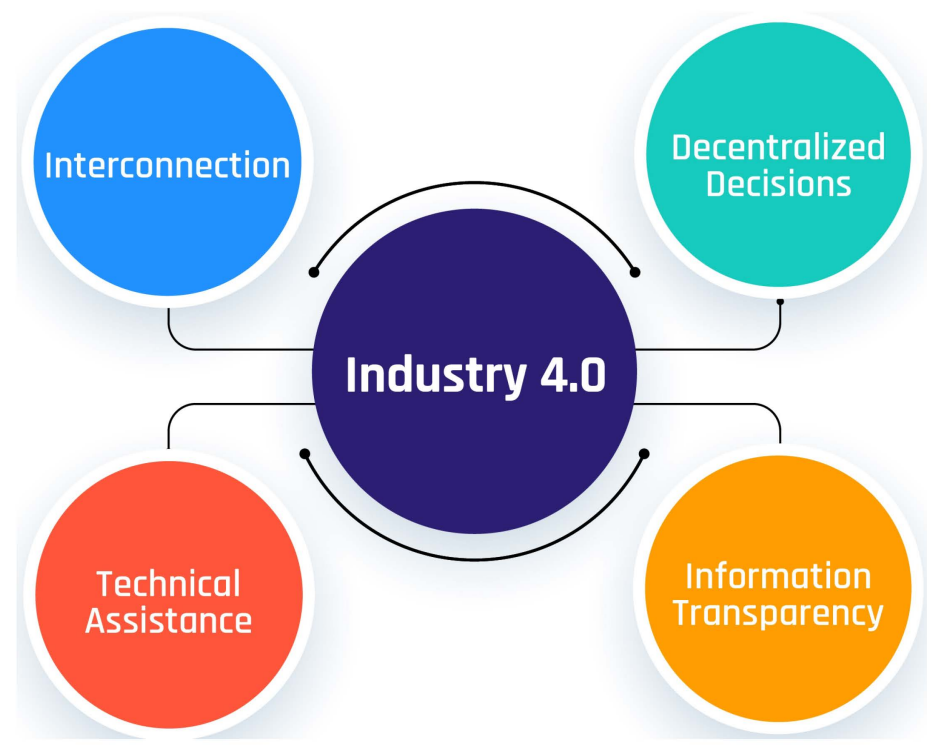

Figure 2. The four main principles of Industry 4.0.

The four design principles of Industry 4.0 are as follows:

- Interconnection: Interconnection is the ability of all the available components to communicate and connect. These components include devices, sensors, and machines. They can connect with people operating them through the technologies related to Industry 4.0 [69];

- Information Transparency: Industry 4.0 enables transparency, which provides the operators with comprehensive information that allows them to make informed decisions. The inter-connectivity of all the systems allows the operators to collect data and 
information from all sources. They can use these to make the manufacturing process more efficient and identify critical areas where improvement can lead to increased functionality [69];

- Technical Assistance: The systems should have the technological facility to help the operators in any capacity. These can be for decision making, performing unsafe tasks, and problem solving [70];

- Decentralized Decisions: The cyber-physical systems that form Industry 4.0 should be as autonomous as possible and have the ability to make their own decisions and perform tasks themselves without requiring any assistance from an operator. Tasks should only be reserved for the operators at higher levels when there are interference, conflicts, and exceptions [71].

\section{Industry 4.0 for Medical/Healthcare Services}

A significant amount of studies have been conducted from different perspectives in healthcare sectors [72-74]. Industry 4.0 enables technologies to make the healthcare sector more straightforward than before; for example, improving and enabling remote monitoring systems based on IoT. In addition, measuring any kind of quantity within the shortest possible time, cloud-based record systems, and fast communication are some of the most noticeable advancements. Transferring data from one place to another is another advantage of Industry 4.0 technologies [11]. The technologies involved in Industry 4.0 can reduce associated costs and time [75]. In addition, healthcare support can be provided instantly with the help of such technologies. Technologies such as IoT, blockchain, Cloud Computing, and AI have led to some fantastic improvements in healthcare. The role of Industry 4.0 in healthcare is extraordinary, as it has reduced time, costs, and led to the implementation of better solutions [76].

\subsection{IoT and BDA in Healthcare 4.0}

IoT has gained a lot of attention, due to its deployment in different sectors, and can be beneficial in areas such as wastewater treatment $[77,78]$, climate study, remote sensing [79-82], smart grid security, healthcare, and so on. In the healthcare sector, the "digital data foundation" consists of modern technologies, including IoT, which is also one of the fastest adopted technologies at present. It describes remote monitoring services. Remote patient monitoring devices are basically a core digitized technology to send and receive information between patients and medical facilities. The patients can monitor themselves and collect data then, with the help of IoT devices, the data can be electronically transmitted to physicians. IoT is a necessary element of Healthcare 4.0, which has led to remarkable changes in the healthcare sector. IoT operates to identify, detect, and authenticate intention and people for special medical services.

\subsubsection{Applications of IoT and BDA in Healthcare 4.0}

IoT is a widely used name in Industry 4.0. Present IoT implementations are quite large. There was a time when communication was slow and proper networks were lacking. IoT solved these problems with great merits. It refers to many physical devices around the world, which are all connected through the internet, in order to collect and share data. The network is connected with wireless sensors, and shares and collects data to provide the desired results.

In the healthcare sector, IoT has a huge impact. The modern healthcare sector can also be said to be part of Healthcare 4.0, a branch of Industry 4.0 [14]. Healthcare 4.0 consists of different applications. In healthcare, the term "digital data foundations" relates to the fastest adopted technology, IoT, and describes remote monitoring services; in most cases, for patients. There was a time when scheduling an appointment with a doctor was problematic, and the patients had to go through a long wait period to meet a doctor. Sometimes the patients had to face difficulties during situations where they needed immediate medical attention, such as breathing difficulty, chest pain, and haemorrhage. 
IoT in digital healthcare refers to affordable, cheap, accessible, and personalized services. Figure 3 represents different types of IoT technologies applied in Healthcare 4.0.

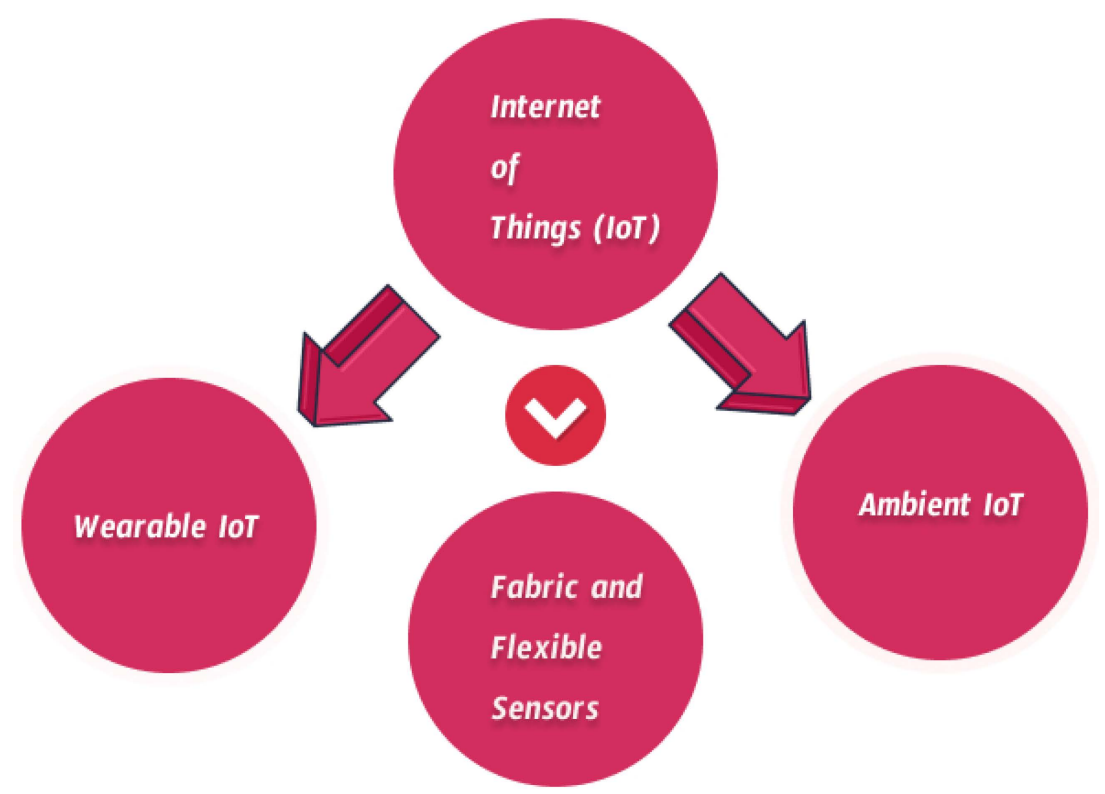

Figure 3. Different types of IoT Technology for Healthcare 4.0 [14].

Examples of wearable IoT devices include smartwatches (Apple Watch, Amazfit) and smart glasses (Google Glasses, Hololens). Smartwatches can display the wearer's heart rate, pulse, steps taken, and much more. These devices are built with wireless sensors, which send data to a central server. The server then displays the results that the individual wants to know. Low-cost disposable patches based on IoT, generally worn for a couple of days, are known as fabric and flexible devices. Biostamp, iRhythm, BP monitor, UV sense, and electro pads are examples of flexible disposable patches. The Australian Centre for NanoMedicine (ACN), along with the University of New South Wales Sydney (UNSW), have developed a wearable sensor that informs the wearer of their exposure to ultraviolet rays [14]. Ambient IoTs describe non-wearable IoT devices, such as motion, door, pressure, video, object, and sound sensors. Physicians can directly access their patients through remote monitoring with ambient IoT devices. In industrial scenarios, extending IoT has had a significant impact on the healthcare sector. Industrial IoT industries incorporate networks of connected devices [83]. These connected devices share data, while data analysis for the system consists of collecting the data and generating results.

According to Lu, Papagiannidis, and Almanos (2018) [84], the term IoT is not as simple as fundamental ideas or knowledge. As it connects various things and objects in a network, IoT is based on three visions: Internet-oriented, things-oriented, and semantic-oriented. In the healthcare supply chain sector, the IoT has been used for a long time. Applications related to the healthcare supply chain are designed to track, identify, and collect victim, patient, and staff data. As is well known, IoT-related devices are attached to sensors, which continuously capture and collect data about the patient. These data include health indicators, blood level, heart rate, and much more. After obtaining real-time data, it is transmitted to a medium device, which can be a computer or a device, through a wireless network. The data are then analyzed. By using a wireless system, IoT saves the time and effort of medical staff. IoT can improve the quality of healthcare services and lower their cost. The second device, known as a remote control, allows for excellent communication between physicians and patients. The physicians can communicate and judge the health condition of patients, as well as give suggestions through remote control. The position and status of patients can be monitored by Radio Frequency Identification (RFID) and infrared 
data association technology. Similarly, Big Data enables healthcare applications to explore new insights and find accurate solutions [85].

\subsubsection{State-of-the-Art of IoT and BDA in Healthcare 4.0}

IoT enables the use of remote monitoring systems, which has a huge positive impact on healthcare. Health-sensing devices have been developed at a whole new level to measure the heartbeat and blood pressure of patients, among other things. State-of-the-art technologies in IoT are numerous. As a result of the fourth industrial revolution, the technologies used in healthcare are remarkable. Existing technologies are used rapidly in healthcare. The IoT healthcare network can display the process. The transmission and receiving of data are carried out through the IoT network. IoT healthcare consists of the topology, as well as the architecture and platform [17]. The topology allows for various use-cases, application scenarios, and activities. The healthcare architecture shows the software organization of the system as a whole and reflects the hierarchical model [14]. Library, framework, and environment comprise the platform sector. Wearable remote healthcare monitoring systems are an existing technology used in the healthcare sector. IoT devices are made with sensors, which can provide exact data. These wearable devices (e.g., smart bands and smartwatches) can be used to track chronic diseases. AI-based data processing algorithms can be used to detect data from ECG sensors. Smart bands, such as wristbands, can track footsteps, heart rate, blood pressure, calories, and many more. As such, health abnormalities can be detected through the use of IoT devices.

Mobile smartphone applications, such as mHealth apps, are available in the Apple and Google play application stores, providing information about the health condition of the user. These applications operate by using the sensors available in the phone. Another application area is medication adherence however, this area is expensive and poses a problem for patients, physicians, and healthcare service providers, as it is not always affordable. Some systems for healthcare in IoT have been proposed. One of them is an architecture called a Smart Hospital System (SHS), which is based on an architecture called 6LoWPAN. 6LoWPAN is an acronym standing for IPv6 internet protocol over wireless personal networks. A SHS can collect data in real-time in environmental situations, including the physiological parameters of patients. This whole system indicates an architecture with novel functionalities. This system collects data and delivers them to the main control center, while the monitoring application analyzes the received data and sends alert messages in the case of an emergency [86]. An RFID-enhanced door can operate as a 6LoWPAN Border Router (6LBR). A 6LBR can generate a connection between Wireless Sensor Network (WSN) nodes and other remote users. Here, a WSN can be described as a self-configured and infrastructure-less wireless network, which is able to monitor physical and/or environmental conditions [87]. The monitoring application receives and stores the data in a database. The REST web-based paradigm can be used to easily access local and remote control. A website-based Graphical User Interface (GUI) enables and allows network and communication operators to manage the environmental parameters of sensors [17].

A cloud-based IoT architecture, which we call "secure cloud-based IoT framework for neuroscience applications" [18], has been proposed as a model for neuroscience technology. Big Data and the cloud are the foremost products for the development of collaborative frameworks for defragmenting brain signals; for example, Electroencephalograms (EEGs), ElectroCochleoGraphy (ECoG), Anteroposterior (AP), Local Field Potential (LFP), and so on. Magnetoencephalography (MEG), Magnetic Resonance Imaging (MRI), Functional magnetic resonance imaging (fMRI), and Positron Emission Tomography (PET) can be used to capture brain imagery. These can be used, together with fog computing and edge computing, to deliver the necessary medical procedures to patients remotely $[16,88]$. A secure cloud-based IoT framework for neuroscience consists of three main components: The IoT end, the cloud component, and processing and analysis. The framework shows that various neurotechnology enables devices to collaborate in developing state-of-the-art techniques which are effective for neuroscience and healthcare research. In the perception 
layer, various types of data-propagating devices are attached to the data receiver. The receiver dedicates data to the cloud through an implemented IoT pathway [18]. Brain signals can be used to generate IoT responsible for maintaining various medical and effective apparatuses, in order to provide better understanding and monitoring.

In a cloud-based IoT system, the sensors (or IoT engines) produce data associated to different neuroscientific partitions. Similar to the relationships in humans, these sensors collaborate and define such patterns. At the time of different interactions, the trusted metrics of every node are merged through a neuro-fuzzy method [18]. The mixture of physical and computational methods is known as cyber-physical systems. Cyber-physical systems generally describe robots and sensors [89]. The frameworks followed by IoT and data services are related to cyber-physical systems [90]. The concepts of bio-sensors, simulated organs, and smart pharmaceuticals are familiar as aspects of science fiction from the past [91]. At present, with the invention and development of IoT, such fiction has become reality.

Different agendas have focused on electronic-based projections. The next concept is the concept of smart factory management. Smart factories can be called the heart of Industry 4.0. Industry 4.0 ensures the ability to automate tasks and help people to reach their engaged task goals. In addition, Industry 4.0 has introduced context awareness [92]. With the rapid progress of IoT, data for patients have been piling up rapidly. In terms of improvements to ensure secure databases, cognitive systems help in predictive prospects. Cognitive system algorithms can use self-learned data with the help of deep learning. ML and pattern recognition use cognitive system algorithms [93]. Most of the time, the algorithm is content-based but, in some cases, it consists of collaborative filtering in nature. Such algorithms can be model-based or memory-based. e-Health and m-health are two principles of CPS, where the mixture of information and communication technologies are known as e-Health, involving such tasks as collating insurance coverage data. On the other hand, m-Health can be stated as an example of Industry 4.0. ML concepts can be operated in any advanced or smart environment. At present, most industries are pursuing the "smart factory" notion, which is prioritized through automation. The influence and effect of Industry 4.0 are huge, which has become widespread in the field of engineering, in order to produce smarter and intelligent robots for better and rapid results, among other uses.

Big Data generally indicates a large volume of data, which can be structured and/or unstructured. Big Data is characterized by five properties: Volume, velocity, variety, veracity, and value [11]. A present source of Big Data is enterprise data. Enterprises produce and manage high volumes of data, being responsible for storing employee data, internal communications, and accounting. It is also applicable for healthcare billing, administration, and scheduling information. Big Data use-cases in healthcare can be divided in categories, including consumer behavior, administration and delivery, clinical decision support, and support services [94].

\subsection{Blockchain Technology in Healthcare in Healthcare 4.0}

A blockchain is a distributed public ledger, guaranteed by a peer-to-peer network that records transactions and tracks assets with no centralized authority. The blockchain's records are arranged in an orderly block structure. These blocks are chronologically connected; this whole chain is called a blockchain. In this design, no block from the middle of the chain can be altered without altering the other blocks simultaneously. This characteristic enables blockchain networks to be immutable. This immutability of blockchain data is one of the main aspects of why blockchain has drawn attention for use in the healthcare sector. Blockchain is primarily expected to improve medical record management, insurance claims, and to advance clinical and biomedical research [95]. The main aspects of blockchain, such as data provenance, robustness, decentralized management, security and privacy, and the immutable nature of the stored data, are key factors that have been considered in order enhance the data situation in health and medical services. One key attribute of medical data 
is that they should be possessed and utilized by the subjects who provide the data and not hold the data. Blockchain technology can enable the recovery of the subject's rights to their medical information. To date, no requirements have been listed for a blockchain-based healthcare management system. A list of seven criteria for a management system using blockchain has been proposed by [96], as shown in Figure 4.

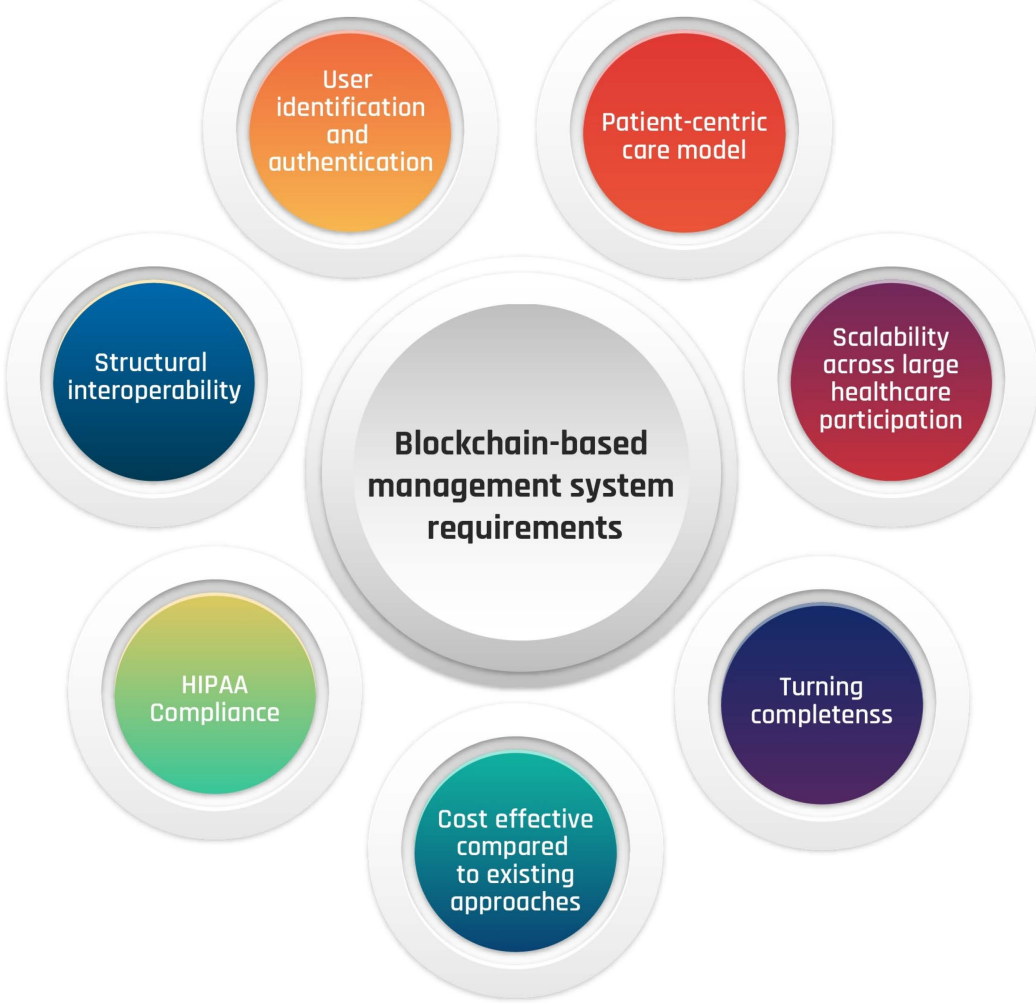

Figure 4. The seven requirements for a blockchain-based management system.

\subsubsection{Applications of Blockchain Technology in Healthcare 4.0}

For healthcare/medical services within Industry 4.0 using blockchain technology, we analyzed three papers [97-99] written with these specific topics in a correlated manner. The existing methods for handling the security of Electronic Health Records (EHRs) make these records inaccessible to patients [98]. Researchers have suggested a blockchain-based framework, in order to maintain and efficiently store EHRs while enabling efficient and secure access to the data and preserving information confidentiality. The data can be accessed by patients, third parties, and health providers through the framework.

Blockchain may be a viable solution in Healthcare 4.0 applications. Places where middle-men are involved, such as claims and billing, can be a source of data leaks in hospitals. Medical research is currently conducted in local systems, hampering efficient research sharing. The security of patient data can be compromised due to data generators and the multiple devices required for the end user. In Drug Supply Chains, the patient profile needs to be accurate to deliver drugs efficiently and deter counterfeits [42,100]. For the author's part [98], they proposed an approach that stores and transfers EHRs using blockchain. They analyzed how the proposed system can fulfill the requirements and showcased how the system will maintain the privacy and security of patients, third parties, and health providers under the Healthcare 4.0 paradigm. They also discussed functions that enable blockchains to be effective and discussed other contracts in Healthcare 4.0 [98]. Their proposed framework was divided into execution and communication modes. Execution occurs as a call, which is operated upon at the end-user's local machine, but only works on a read-only paradigm. They do not go through the network and, hence, do not need to be authenticated [101]. Communication is carried out in two ways, where the 
contracts communicate through inter- and intra-networks. One contains messages, in which contracts are sent between themselves, which are initiated from a single transaction [101]. The IT components require database managers, which navigate the saved records of the patients, and cipher managers, which handle the cryptographic requirements of the paradigm, managing encryption and decryption for stored files. Blockchain contracts are part of the nodes that constitute the chains. This blockchain contains the classification contract, consensus contract, service contract, owner contract, and permission contract. The architecture of the system performs the functions shown in Figure 5.

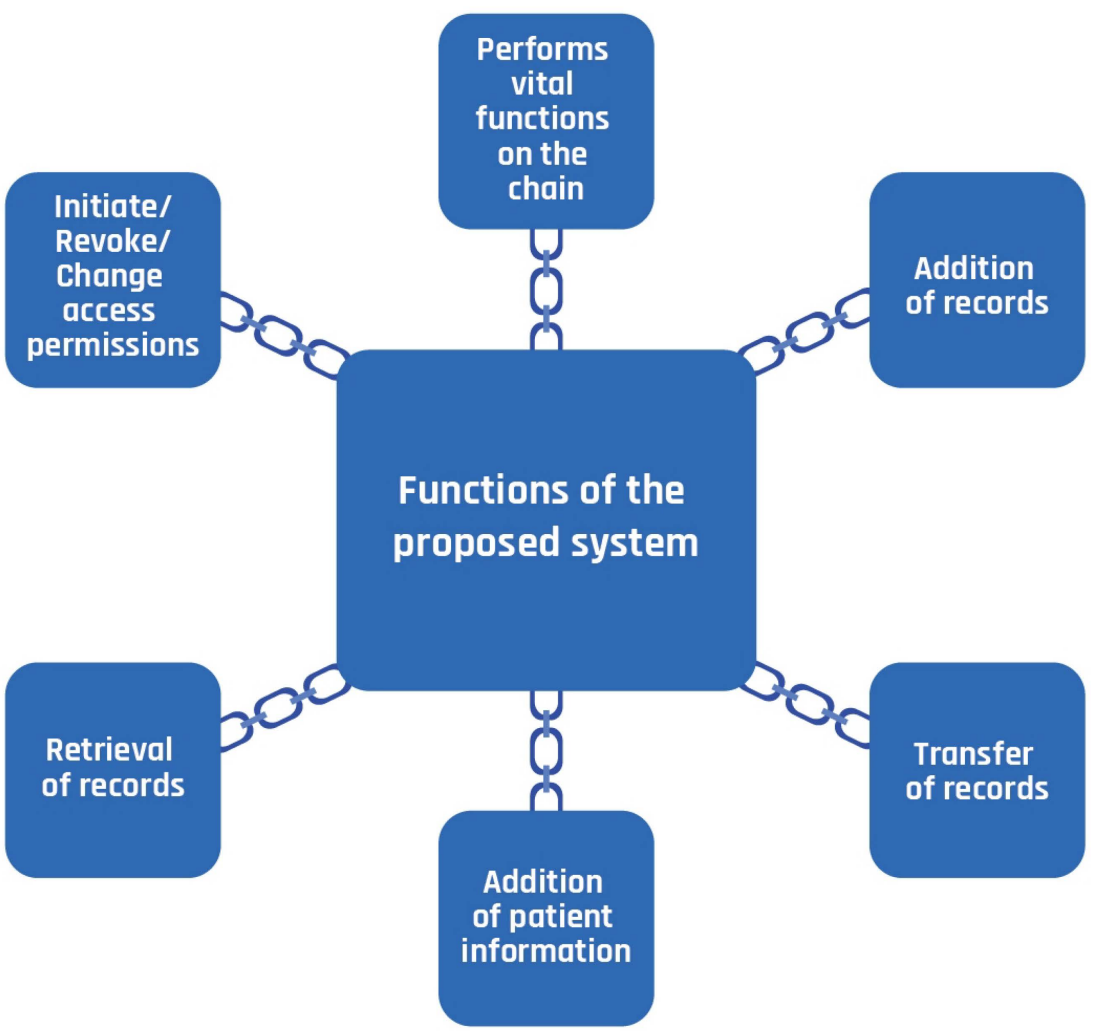

Figure 5. Functions that can be carried out by the proposed system [98].

Expensive and complex health-related modern systems can be improved using blockchain technology, with better utilization and proper record management of health information [99]. The researchers provided several solutions for improving healthcare systems with blockchain, using frameworks and tools to measure performance in order to overcome the limitations that are currently present. The systems they mentioned include wireshark, docker, and hyperledger fabric. An algorithm was also proposed to enable easier access to data for health providers. The most significant difference in this paper was their proposal for blockchain to be used to improve security and efficiency in existing client- and server-based systems.

Top-level privacy and security are required for Healthcare data and, with the inception of Healthcare 4.0, the dependence on smart technologies has increased worldwide. Big Data has caused healthcare records to increase in complexity and size, while they remain unoptimized. Considering the trusted, distributed, and immutable manner in which data are stored and shared, the research popularity of smart ledgers and blockchain has increased. Blockchain offers integrity and improved verification of data generated in healthcare settings, and helps to distribute the data in the network. This open and decentralized system eliminates the middle-man from the system, which means there is no requirement for multi-authentication and provides data access to anyone in the network [102]. The authors contributed by proposing a patient-centric approach that provides access control using distributed ledger technology, in order to provide information 
to different healthcare providers using symmetric key cryptography. Their proposition is an approach using blockchain which implements information sharing through the concept of permission-based chain code. They have determined the best approach for the blockchain system's performance optimization metrics, with regards to throughput, latency, security, and work scalability.

In the blockchain network, an EHR is distributed to others in the network through a shared private key and a symmetric key in the proposed system. There are four participants in the proposed system: Patient, lab, clinician, and admin. Participants register to the system through a Membership Service Provider from a certificate authority. Then, they can access records that they have been granted permission to, and the service providers can update new records to the participants after they have been permitted to update the records. All of the records are visible to everyone on the blockchain. If anything is updated on the blockchain, it is distributed to everyone on the network, making the network secure and preventing the modification or deletion of records by unauthorized users. There are four different types of executions that can be made in the system-for the admin module, patient module, clinical module, and lab module-the workings of which have been detailed previously [99]. Their electronic health record system was developed using a hyperledger fabric framework and hyperledger composer sandbox. To use these, they utilized an operating system-level container, "Docker", which is a set of platforms as a service product that uses OS-level virtualization to deliver software packages, in which the packages are known as containers. Visualization of the network and the implementation of multiple applications are supported by the frameworks. The blockchain is permissionbased, and a consortium is managed to allow all the participants to be known to each other, making it secure and trusted. The framework not being domain-specific allows for the support of other domains. They evaluated their system's performance with varying parameters, including creation time, block size, and endorsement policy. They displayed the results based on throughput, performance latency, and network capturing. Their results showed that committed transaction latency does not depend on block size. They achieved even better latency results when they lowered the transaction rate while configuring block sizes. Ultimately, they found a smaller block size with lower transactions and larger block size with higher transactions resulted in better performance. The authors outlined how critical the role of blockchain is, with the automation of data collection and verification process in the current healthcare market, as well as providing a reduction in crimes and security of data.

The value of medical information is higher than even financial information to criminals, and information related to health has been shown to be more vulnerable in current Healthcare 4.0 systems [97]. The researchers explored the use of blockchain technology, in order to show its advantages when used in Healthcare 4.0 scenarios. Industry 4.0 has started to expand through more modern technologies, with Hospital 4.0 also following suit, in which the Industry 4.0 paradigm is applied to the healthcare industry. As an emerging technology for data management, blockchain can mitigate some of the threats that data storage and management face in general. With cryptography and distributed consensus ensuring security, integrity, and accountability, blockchain technology can provide an effective solution to the modern and future Healthcare 4.0 scenario.

Industrial IoT (IIoT) has extended the IoT paradigm into mainstream industries, incorporating connected devices into networks and, thus, forming a data generation and exchange system. Data Analytics systems collect valuable data from the whole system, generating insights which allow entities to make smarter and better decisions; for example, relating to predictive maintenance, asset tracking, and facility monitoring. Hospital 4.0 is another iteration of Industry 4.0 within the same paradigm. It enables cooperation and communication between humans, network services, IoT devices, and cyber-physical systems, in order to enable healthcare services [103]. The requirements to fully adapt the Healthcare 4.0 paradigm have been summarized into the three main points, as shown in Figure 6 [97]. 


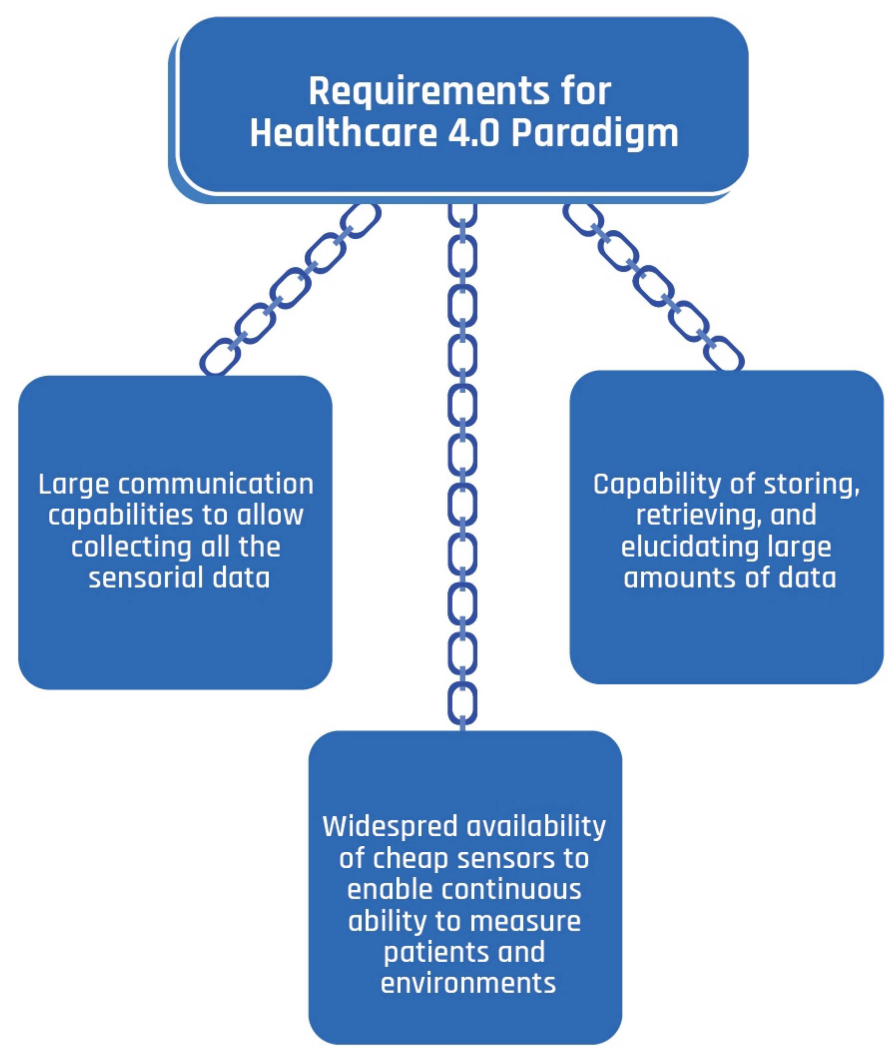

Figure 6. Requirements for full adoption of the Healthcare 4.0 paradigm [97].

Hospital 4.0 has four main principles that it needs to cover, in order to be eligible for use in Healthcare 4.0 [97]. EHRs, which increase the convenience of information sharing, are also required to conform to certain parameters, in order to be eligible for implementation in the system [104]. These are shown in Figure 7 [105-107].
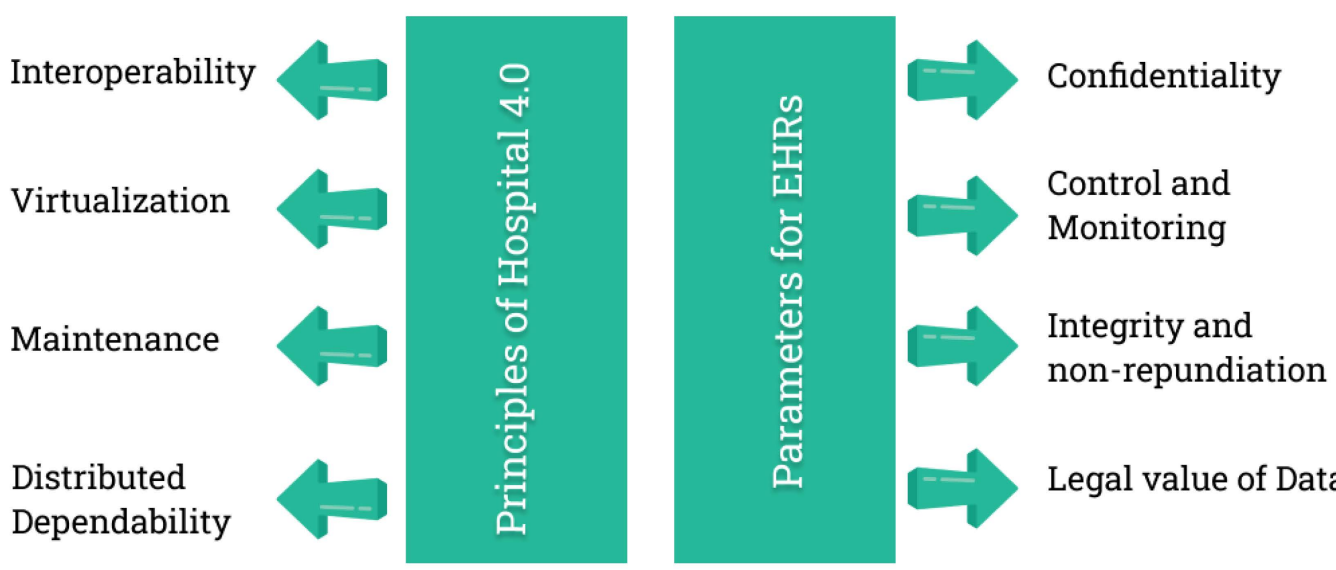

Figure 7. Principles of Hospital 4.0 and parameters for EHRs [105-107].

\subsubsection{State-of-the-Art of Blockchain Technology in Healthcare 4.0}

SimplyVital [28] is a protocol based on blockchain that enables secure healthcare adoption. A New England-based business created Health Nexus, a healthcare-related blockchain ecosystem. Their vision is to enable everyone to access healthcare data easily and securely. Their task is to enable data access securely through a protocol that is HIPAA compatible with a care network using blockchain [29]. MedRec uses blockchain to handle EHRs in a decentralized system that manages the records [32]. Patients are provided a comprehensive $\log$ of their information, with ease of access, from their medical professionals. MedRec [33] 
manages authentication, accountability, confidentiality, and data sharing of medical data using blockchain technology. The system's design is modular, enabling it to be integrated with existing systems from the provider's local data storage systems. The medical stakeholders (i.e., researchers, health authorities, and service providers) are incentivized to take part in the network with proof of work. This blockchain gives them access to anonymized and aggregated data on the network. Their goal is to enable the emergence of significant data economics and supply data to better enable researchers, while allowing the providers and patients the choice to use their data. Zhang et al. [34] considered a healthcare system that can share and authenticate data in a network based on Policy Service Nodes (PSNs). They proposed two protocols, one of which uses blockchain-based health information sharing among PSN nodes. In one of their use-cases, they proposed human body-based channels for PSN nodes. Medicalchain, a platform that can store and share health information that were previously authorized based on a blockchain, can facilitate the immediate retrieval of health records, which allows patients to communicate directly with doctors and share their health records online instantly for consultation. This is expected to enable the future of accessible healthcare, with safe and secure online consultation. Enabling patients and doctors to have secure access to healthcare records can create an empowering position for both [30].

Xia et al. [35] used a blockchain and cloud-based approach for sharing healthcare data. Due to current medical data management methods and protection being insufficient, the authors proposed MedShare, which attempts to solve the problem of sharing data in the medical environment. Their system allows data entities to share medical data in cloud repositories with provenance, control, and auditing, based on the use of a blockchain. They introduced a data custodian system which monitors the data being accessed for malicious usage. Any transaction that occurs in the system is recorded in the blockchain for integrity. The design of the system enables access control mechanisms to track data behavior, in order to detect permissions violations. The compatibility of MedShare is on par with current data solutions used by cloud services. Jiang et al. [36] designed a chain verification exchange system for data with blockchain. Healthcare Information Exchange (HIE) has benefited the medical industry with profound effects, allowing for the secure storage of large amounts of data. Their proposed system, BlocHIE, uses blockchain technology to exchange healthcare information. By analyzing different requirements for healthcare data sharing, they used two blockchains to handle different data types of health data that were loosely connected. The advanced blockchain architecture is a new system solution to supply a reliable mechanism for secure and efficient medical record exchanges [31]. The authors proposed this approach to meet healthcare growth demands while creating a new form of interactive social norms. Their goal was to improve patient outcomes through digital health solutions and services, produce less complex e-health systems, decrease costs, and improve efficiency, reliability, and flexibility under the Healthcare 4.0 paradigm. They explored Advanced blockchain in healthcare record storage and data privacy. With scalable and interoperable networks, services, and applications becoming available for electronic health records, the need for robust security and personalized data privacy models is a requirement for consumers. Wang and Song [37] proposed attribute-based record sharing for medical data systems. The medical data are encrypted with identity-based encryption and attributebased encryption. To implement digital signatures, they used Identity-Based Signatures (IBS). As these different cryptosystems are difficult to implement correctly within a single system, they worked around this issue through the introduction of combined attribute/identity-based encryption and signature (C-AB/IB-ES).

Yup et al. [39] designed a data gateway for healthcare. Healthcare data are a very vulnerable source of information, and healthcare intelligence makes it an asset that the asset provider should control. Sharing this information is difficult, as it is typically scattered throughout different healthcare systems, which puts the provider's privacy at risk. Their proposed app, Healthcare Data Gateway (HDG), is a secure and efficient architecture based on blockchain which allows patients to control their data and use it as they want, without 
violating privacy rights. They proposed a purpose-centric access model to enable the owners of data to control and use it in a simplified way through an Indicator-Centric Schema (ICS). Patientory [38] allows users to create and track their health information, while also providing easy monitoring of information. Their goal was to enable people to have control over their health-related information. They are creating a method for doctors and patients to interact and gain access to information, while removing all the layers that slow down the process and reduce efficiency. Their app allows users to securely access, store, track, and exchange their health data with their chosen medical professionals, eliminating many of the stumbling points in the current healthcare environment. Posted and Poslad [40] discussed electronic record access control with fine granular access. Existing blockchain schemes cannot authorize different levels of granularity of authorization. For this, they proposed a system: Granular Access Authorization Supporting Flexible Queries (GAA-FQ). Regular blockchains require public key infrastructure (PKI) to access the data however, GAA-FQ does not require it, due to their authorization, encryption, and decryption algorithms, which enhance the computation performance and enable more granular, distributed support for authorized EMR data queries. They benchmarked GAA-FQ against ESPAC, another access control scheme, in order to validate its computational performance and transmission efficiency.

EncrypGen has provided a genomic data sharing platform. Their Gene-Chain platform is the world's first fully functional blockchain genomic data marketplace. Generally, consumers can provide their DNA samples and have information extracted from them however, this creates privacy issues. With Gene-Chain, the participants can have their data stored anonymously and the blockchain enables it to be secure [41].

Liang et al. [42] introduced a blockchain-based system for mobile systems, designed to share health-related information. Personal health data are now more accessible, thanks to wearable technology, which has increased its value, benefiting both medical research and healthcare providers. The proposed system is a user-based health platform, where data sharing is conducted through a blockchain to protect the data. The protection of information is ensured using channel formation schemes. The data are collected through a mobile application, from the user's wearable devices, medical devices, or manual data entries. The data are synchronized on the cloud, where they can be shared with professionals, medical research, and insurance entities. The integrity of the data is preserved with validation records that can be retrieved from the network, which also incorporates a cloud database.

Coral Health provides a medicine system that is personalized to patients using blockchain technology [43]. Although EHRs have enabled the sharing of records, they remain costly. Coral Health utilized an approach to introduce a new app which makes it possible to integrate medical records with the app. The stored data, under HIPAA compliance, is stored in the user's smartphones with total control over the encrypted data [44].

Chronicled is a solution using IoT and blockchain in order to increase the accountability of supply chains in life sciences. A network called MediLedger Network, powered by blockchain and led by the life sciences industry, comprises their main contribution. The complex industry, where pharmaceutical drugs move from manufacturing to serving the patients, features many regulatory requirements, contracting relationships, complicated policies, and a large number of exceptions to industry standards. Navigating this complex track is very difficult, but blockchain presents an opportunity, where every company has influence over their data and the transactions that they receive from their partners. This enables secure automation in the industry, beyond internal systems [45].

Thakkar et al. [46] provided two approaches to analyze the performance of systems using blockchain. One popular approach is the use of Hyperledger Fabric permissions blockchain platforms hosted by the Linux Foundation [47]. Hyperledger Fabric has been comprehensively studied, in order to characterize its performance, identify its possible bottlenecks, and determine ways to improve the overall throughput. Their first task was to identify the impact of various configuration parameters. State validation, endorsement validation, and transaction policy validations were identified as the three main bottlenecks. 
Secondly, they optimized the Hyperledger Fabric framework by parallel verification and aggressive caching. Another optimization they carried out was bulk read/write optimization during state validation and commit phases. Combining all of these, they reported a $16 \times$ overall throughput improvement. Sukhwani et al. [48] carried out an analysis of performance metrics of the hyperledger fabric framework. With blockchain networks bringing benefits to networks with large peer numbers, such as EHR networks, their performance must be better than conventional IT systems in order to justify implementing them at a large scale.

The next study focused on the roadblocks that prevent blockchain and EHR systems from becoming mainstream [49]. The solutions proposed by the authors, in terms of their platform for sharing data based on blockchain, attempts to address the problems which are crippling for blockchain, in terms of sharing data. Their proposed design uses a distributed microservice architecture which allows for the encapsulation of functions that are integral to the system, as well as services that are independent and isolated, with requirement-based scalability for different systems. Their solution is containerized, guaranteeing simplified installation, portability, and reduced overhead cost. Another implementation is focused on sharing EHR data, as implemented at Stony Brook University Hospital [49]. EHRs contain highly sensitive data, and they are shared frequently among healthcare service providers and service seekers. Blockchain has been implemented to enable trust, transparency, and accountability for the network with sensitive data, and provide a transparent, immutable, and shared platform for data transactions. Their frameworks to share and manage EHR data consists of two approaches. One of the two suggested approaches is institution-based, where the network comprises trusted service providers and patients (from whom the data are collected). The other approach is case-specific where, for the hospitalized patient, a network that connects doctors, nurses, and families is envisioned, in order to reduce mistakes and increase the likelihood of effective treatment. At present, this proposed system's goal is to significantly reduce the time it takes to share EHRs, reduce overall costs, and improve decision making for medical care. Gorenflo et al. [50] put forward a redesign of modern permission blockchain systems to optimize and increase the throughput to 20,000 transactions per second. The authors redesigned Hyperledger Fabric, a modern permission blockchain system, to increase the transaction throughput. Their main focus was on bottlenecks related to performance, with proposals for change in architecture to overcome the bottlenecks. This redesigned version was called FastFabric. They also noted that their optimizations do not require any interface change to the existing framework, and can be implemented in any system using the aforementioned framework.

Uddin et al. [51] proposed a patient monitoring system that would remotely monitor patients in a continuous manner however, due to Remote Patient Monitoring (RPM) requiring large sets of data and power, it is challenging to implement. The authors proposed the implementation of a patient-centered architecture that is end-to-end, based on tiers. The final implementation mentioned is ModelChain, a decentralized predictive modeling healthcare framework using blockchain that preserves privacy [52]. Predictive modeling for healthcare that is shared between institutions can enable better quality research. Predictive models that protect privacy do exist, but they present security and robustness vulnerabilities. Participators contribute to the model by exchanging data, and no private information is revealed. An increase of interoperability between institutions is one of the benefits of such an implementation.

\subsection{AI in Healthcare 4.0}

The implementation of $\mathrm{AI}$ is widespread, including in electricity infrastructure, education, industry, and so on [108-116]. Similarly, in the healthcare sector, there has been an enormous contribution of AI. The aftermath of the implemented applications has had a remarkable effect on healthcare. 


\subsubsection{Applications of AI in Healthcare 4.0}

The study [55] reviewed healthcare applications in Industry 4.0 to fight the COVID-19 pandemic, with proper technologies including AI. The detection and diagnosis of COVID19 has also been discussed. This paper attempted to combine the benefits offered by Healthcare 4.0 and its different technologies during the COVID-19 pandemic. Roboticsbased treatment can be conducted on patients using AI to reduce the risk for doctors through the use of virtual reality [117-120]. Technologies used in Healthcare 4.0, such as AI, can detect any problems that the patient is going through immediately, through the use of sensors, in an emergency situation. At present, Healthcare 4.0 technology is being used for AI-based video monitoring in China, helping to observe the activities of patients affected by COVID-19 and to help stop the spread of the virus [53]. Using AI-based technologies, clinical trials of drugs and vaccines against the virus can be optimized [55]. AI has also helped to manage supply chains in the medical sector during the pandemic [121]. AI can be used to perform CT scans, in order to detect pneumonia caused by the virus. In this lockdown situation during the COVID-19 pandemic, an AI-based robot can act as a police officer, ensuring that people are following lockdown protocols. AI can be used to monitor human gatherings, which can then be used to regulate human interaction levels during the pandemic [122]. Even during a pandemic, health data security is important and must be preserved. Fortunately, AI can enable that to be done remotely [123]. Figure 8 highlights some of the uses of AI in the fight against COVID-19.

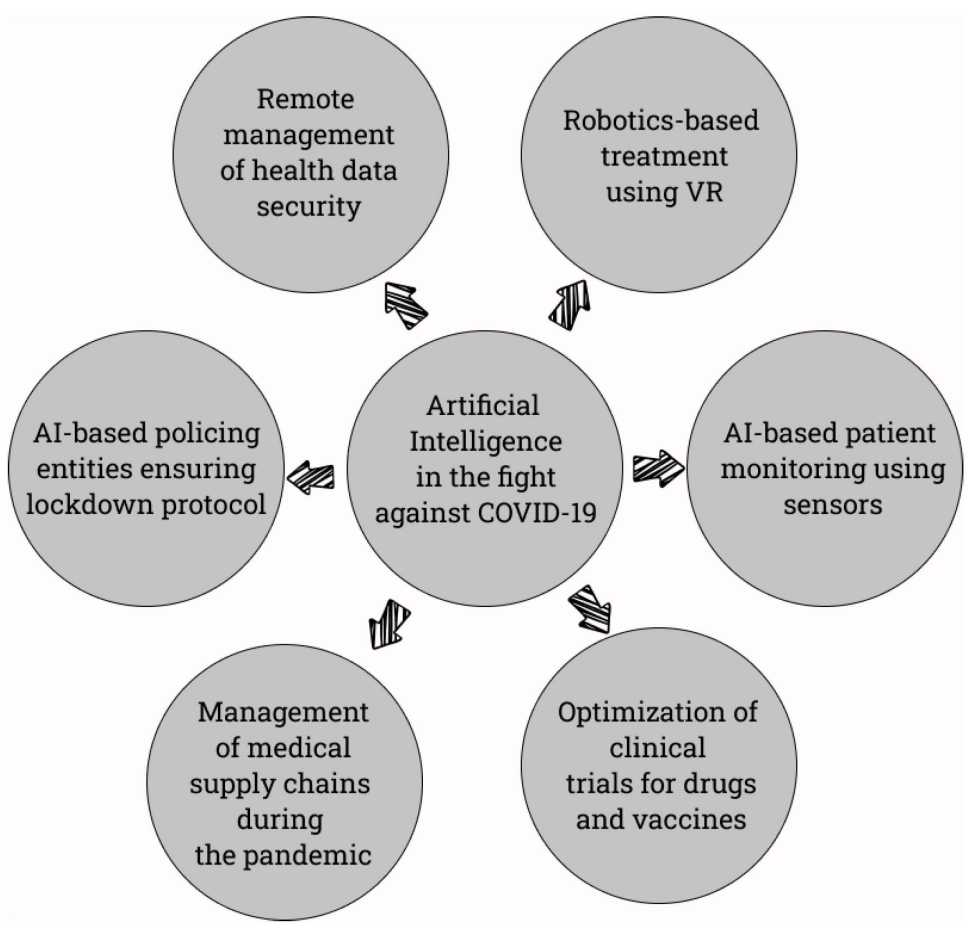

Figure 8. Uses of AI in the fight against COVID-19 [76].

In many organizations in different countries, the health information of individuals is stored and transmitted when needed. Privacy prevents unauthorized access to an individual's information. Under Healthcare 1.0, IT frameworks were important. The second era, known as Healthcare 2.0, required quick storage access. To access data from EHRs, WDs were used. The concept of Healthcare 3.0 started from here. The fourth industrial revolution led to remarkable changes in the healthcare sector, known as Healthcare 4.0. Healthcare 4.0 combined all the previous technologies and incorporated IoT, cloud, and blockchain techniques. Using WDs, IDs in the system (known as a telehealthcare system) to develop traffic between patients and organizations of doctors could lead to a new trademark [124]. It is not easy to ensure security and privacy for healthcare. For this, some 
protocols or requirements must be designed. The mutual authentication involves Kerberos authentication, while anonymity involves hidden addresses or the secrecy of data. If an attacker somehow obtains data, it can be hampered or used in destructive ways. During the login phase, the data or information of both the doctor and patient should be verified. A key agreement or password-protected system could be much more effective to ensure safe login. Untraceability indicates an unrecognizable IP address. The attack-resistance phase indicates fighting against the attacker [125]. Due to the vast amount of data involved, it is crucial to keep the data safe. Security techniques are used to maintain the authorization of an individual's record, with the main aim being to protect it from unauthorized access. By using operational controls, security can be achieved [126].

\subsubsection{State-of-the-Art of AI in Healthcare 4.0}

As the implementation of AI-based technologies has been constantly improving, healthcare AI has had a significant impact on Healthcare 4.0. State-of-the-art features have been enabled in the biomedical sector. In other words, the use of AI is common for Biomedical data types. Multi-omics data consist of genomics, proteomics, transcriptomics, epigenomics, and microbionics [127]. These are collected together and analyzed. The same related biological processes can be characterized by individual omics data sources [128]. The integration of data utilizes a combination of the different views that the data are taken from, from which a single model can be created. The successful implementation of this approach has resulted in the integration of data from mRNA and single-nucleotide polypmorphisms, creating a model named a Bayesian integrative model [129]. The next approach is model-based integration. Each data view is used to build a separate model, and model output aggregation is conducted following [127]. During the integration process, the neural networks and models are first built or constructed, based on each omic data type. Different types of conventional weighted networks have edges enriched in contexts related to multi-omic data. For the analysis of multi-omics networks, AI methodology development has shown huge potential [130].

EHRs, medical images, physiological signals, and clinical data have been analyzed using AI technologies. These type of analyses are often carried out using a process called feature engineering, a method where descriptors are extracted from images and categorized into different classifications or segments. An EHR is a systematic collection of patient's health information [131]. Structured health information indicates data that contain lexicons involving demographic, medication, procedures, laboratory test, and diagnosis data [132], while unstructured information consists of text-based documents, such as medical notes from nurses and doctors. Effort has been made to improve AI methods for EHR analysis. The ML models to analyze structured information is vector-based [133], where the patient records are collapsed into vectors. It enables the summary of the patient and features to be analyzed in different dimensions. Structured EHR data have been analyzed using recurrent neural networks [132]. Congestive heart failure risk was first predicted using an RNN by Choi et al. [134]. Continuous data sets are categorized as physiological data, taken from processes such as electrocardiograms and ECGs. In recent years, more advanced technologies have been used, such as deep learning by Hannun et al. [135], who detected heart arrhythmia by mapping the EKG signals to a series of rhythm classes in a 34-player CNN model. Schwab et al. [136], on the other hand, tried to conduct the same operation using RNN techniques. Video and conversational data have gained a new place and attention inside and outside healthcare-related fields. The Chinese tech giant Tencent has developed and improved a system that is able to detect Parkinson's disease using video data in three minutes. Tang et al. [137], based on transcripts from clinical trials performed by Tencent, created a conversational agent which maximized the accuracy of diagnosis of MCI, compared to supervised models, using a small number of conversational events [127]. 


\subsection{Cloud Computing in Healthcare 4.0}

Industry 4.0 also consists of cloud computing, which has applications in the healthcare sector. Cloud computing allows health institutions to store all the data needed to avoid the extra costs of maintaining physical servers.

\subsubsection{Applications of Cloud Computing in Healthcare 4.0}

With the progress of technology in the Industry 4.0 paradigm, cloud computing has led to remarkable offerings in the healthcare industry. Cloud Computing can be defined as delivering on-demand computing services, which vary from applications to storage and processing power. It also runs on a Pay-As-You-Go (PAYG) basis. This is basically a method for payment where cloud computing is charged on a usage basis. No data are wasted in the PAYG method, as users only pay for service procured and ready. There are three categories of cloud computing services, and each is used with a different form of the PAYG model. These three categories are divided into three parts: Infrastructure as a Service (IaaS), Platform as a Service (PaaS), and Software as a Service (SaaS) [57]. In the IaaS, users or customers do not have an exact time or limitation. They pay on a per-use basis, or a monthly, weekly, or hourly basis. Cloud providers sometimes also charge for Virtual Machine (VM) usage. No hardware or software are required or needed in this category. The IaaS vendors include IBM, Hewlett-Packard, Microsoft, and Amazon web services. The PaaS is provided in terms of how much the application or memory is used per hour. Google provides PaaS. In SaaS, the pricing and demand are based on quality, the latest features, and storage capacity. SaaS includes Gmail, Trello, Slack, and Office 365. PaaS consists of Flynn, Cloud Foundry, Heroku, and OpenShift. IaaS includes Stackscale, AWS, VMware (for dual OS), and Azure. Figure 9 represents the cloud service model which includes the services described above.

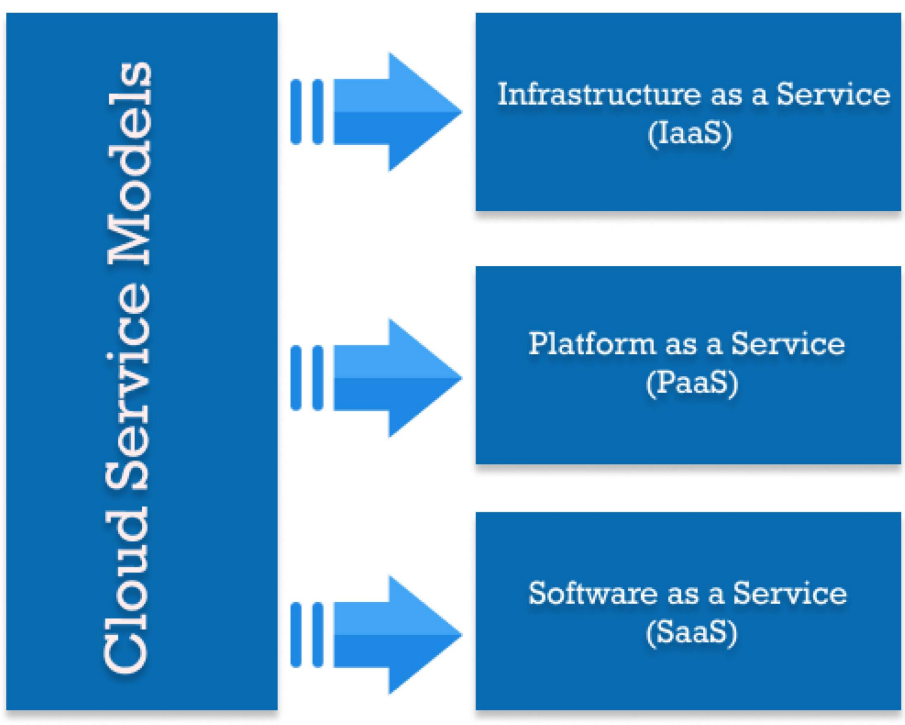

Figure 9. Cloud service models [57].

The most notable cloud computing achievements are limited, focusing on innovative technology. Cloud computing plays an amazing role in maintaining healthcare integration, proposing a new era of innovation. Before moving onto cloud computing, organizations that did not have enough resources were suffering from various problems. At present, it is easy to access anywhere with the help of cloud computing. In healthcare, different information, such as info about patients, doctors, different hospitals, and health organizations, can be accessed from any time and anywhere with the assistance of the cloud. The latest model, which is known as a delivery model, has enriched the healthcare sector, as well as lowering costs. The general proposition of cloud computing is ensuring the storage of huge data and computing power. Medical organizations and healthcare personnel do not 
need to purchase the hardware essentials and servers. No up-front charges are required for the cloud storage of data. Healthcare organizations and hospitals only have to pay for user resources, leading to massive financial savings. The healthcare ecosystem or structure is highly tangled. The complications involve health insurance and hospital-physician networks. Patients also fall under this complication [56] however, there exist some regulations. All work related to healthcare should operate within several government regulations. As for the cloud's help, information should be delivered within the shortest possible time, and there should be security. Securing the data of a patient is the main fact here. The private cloud is applied first, due to security concerns and then it is moved to public infrastructure as a service [57]. The applications include scalable infrastructure, proper data centers, perfect security models, and rapid access to information. The infrastructure and dynamic scalability can be defined as balancing with the growth scenario. As a business grows, organizations invest again to continue the business. For this, a dilemma regarding data storage shortage arises and, for this reason, the resources need to grow as well, as the computing and IT capacities are increasing. With the assistance of the cloud, organizations can have a substitute as well, such that they can trace the capacity issue. The cloud supplies a platform as a service model. On the other hand, it can also provide infrastructure as a service [58], where organizations can apply the current infrastructure and fix or edit the business and needs. Cloud-based data can be used as storage. More servers can be added within the shortest possible time. In the information sharing term, a lot of information is sent and received in healthcare services.

\subsubsection{State-of-the-Art of Cloud Computing in Healthcare 4.0}

Electronic Medical Records (EMRs) are a numeral version of information based on papers in a hospital. EMRs provide and store data about patients (e.g., condition, disease, personal information). EMRs track data over time, and patients can be identified quickly and monitored thoroughly. In a word, an EMR is an electronic record of health-related information where a person or an individual can be created, gathered, managed, and judged by physicians of healthcare personalities within one healthcare organization. According to the health institute, an EMR has five stages: Provider-based computerized medical record, Electronic Patient Record, Electronic Medical Record, Automated Medical Record and, finally, an Electronic Health Record (EHR). The terminologies of EHR and EMR have been used interchangeably by many regiments and medical staff in healthcare industries. To improve the safety and proficiency of patient care, EMR and EHR are both troublesome in the grand vision of healthcare digitization, however, they can reduce healthcare delivery costs [58]. The individual healthcare providers own EMRs. On the other hand, the EHRs are typically composed of some subsets of EMRs. In order to provide high-quality products at an affordable cost, there are no alternatives to EHRs. A Personal Health Record (PHR) is a health record maintained or followed by an individual. Health summary and medical information of a patient or an individual can be determined from the PHR. An EMR is a legal and and accurate data set of a patient, or any staff, regarding the treatment of others. An EMR is generated, applied, and preserved by the merchants of healthcare, in order to look up health data and perform counselling. The patient input and approach can be performed by EHR [138].

\subsection{Start-Ups and Innovative Health Technologies in the Healthcare Industry}

Innovative health technologies and start-ups are essential in bringing new solutions to problems using newer technology and better methodologies. One of them is Adan Medical Innovation SL [139], a company focused on the development of disruptive integrated digital solutions to improve the management of anaphylaxis-a severe allergic reaction caused by, for example, food, drugs, latex, and insect bites. They have created a digital health solution incorporating the current methods on the market—namely, BDA—storing and using essential data about anaphylaxis and their consumers. Their main product is called 
anAPPhylaxis [140], which is a smart case used for Epinephrine autoinjections, connected to a mobile app used by individuals with anaphylaxis.

Qlife Holding is a company based in Denmark who made it easier for patients to measure biomarkers in their blood, using a portable device that can be used at home [141]. This device enables patients to use a home-use kit to carry out convenient diagnostics and monitor blood biomarkers in chronic conditions. Their technology, called the Egoo System [142], includes an optical blood analyzer that takes pin-prick sized samples from patients using a single-use cartridge provided with the kit. The analyzer then detects specific biomarkers, which are determined by the type of cartridge used. At present, they have approval from the European Medicines Agency (EMA) to detect two biomarkers: Hemoglobin for monitoring iron deficiency and c-reactive protein for the detection of arthritis.

Clarigent Health, a mental health technology company, developed a platform that can assist in the early detection of mental health conditions using AI and ML technologies [143]. The platform, called Clairity, acts as a clinical decision support tool providing medical professionals with insights into mental health issues and suicidal ideation. This is done using their patented AI algorithm that detects vocal biomarkers to determine mental health conditions, such as major depression, cognitive problems, and anxiety disorders in people of all ages, as well as measuring suicide risks [144].

Sentio Solutions is a company that has developed biomarkers and digital therapeutics for mental health applications. They have built Feel, an emotion-sensing wristband connected to an app that provides real-time monitoring and personalized interventions to individuals with mental health conditions [145]. They have used their proprietary emotionsensing technology to develop Feel, which tracks changes in the daily emotional habits and patterns of the user using pattern recognition, enabling them to monitor and manage their own emotions and keep track of all of their emotional changes [146].

Bosetein, a German start-up based in Stuttgart, aims to help solve the problem of food safety through their innovation in methods of food inspection using deep learning and artificial neural networks [147]. They developed an optical sensor measuring bacterial contamination in food products in real-time. Their optical sensors utilize AI-based software and can estimate the number of bacteria in real-time, analyze bacteria in real-time, inspect through packaging, and conduct non-contact measurements. The measuring of bacterial contamination on food products in real-time can enable entities to maintain their food products more efficiently and reduce food waste production [148].

Innitius, a start-up based in Spain, uses their patented medical device based on torsional wave technology for the diagnosis of conditions and syndromes [149]. They use a combination of AI and torsional wave technology to facilitate the recognition of Pre-term Labor (PTL), based on tissue consistency-in this case, the cervix. Their product, called Fine Birth, can be used to collect data, in order to find evidence of real labor against false pre-term labor, so they can make clear evidence-based decisions, lower health risks, and improve patient outcomes [150]. With the use of this method, they can make the best decisions towards the end of pregnancies, while also eliminating unnecessary hospitalization and administration of therapeutics.

Evitalz is an Indian healthcare start-up that provides wireless smart medical devices to measure the vital signs of patients, from hemoglobin, ketone, cholesterol, blood glucose, uric acid, and $\mathrm{SpO}_{2}$ to essential parameters such as blood pressure, temperature, and ECG [151]. These are collected securely from the Android or iOS devices of patients, encrypted, and then sent to secure cloud-based information management systems that deliver the information to medical professionals, facilitating decision support and better assistance to patients at home [152]. As a result, patients are able to receive healthcare for their specific conditions from the convenience of their homes, allowing for remote healthcare to be provided securely and reliably.

iSolve has developed an innovative new way of using blockchain technology for the drug supply chain. They have developed Advanced Digital Ledger Technology (ADLT), 
which is a blockchain solution on the enterprise level complementing modern systems and providing security in the drug supply chain [153]. BlockRX is the platform that iSolve uses, incorporating blockchain technology and their ADLT system, integrating patients with life science researchers, medical device makers, healthcare providers, and BioPharma entities. This ecosystem creates new revenue streams and reduces the cost while enabling participants to interact with each other and provide secure access to their data [154].

VAY Sports, a Swiss start-up from a spin-off based in Zurich, has created an AI and computer vision-powered application that detects and tracks people's movements during their workout [155]. The primary purpose of this "digital fitness coach", as they have termed it, provides feedback on the posture of the user during their workout sessions in real-time. A computer model of the user is created through the use of algorithms and computer vision. This is then compared with a model that is created with help from personal trainers to be the optimal model, and provides feedback accordingly [156]. The purpose of this approach is to reduce the risk of injury during a workout and increase user effectiveness.

PokitDok is a healthcare API platform that makes it easier and faster for newcomers to bring in new healthcare implementations of their own. Their main focus was developing APIs for identity management, pharmacy, and claims for the healthcare sector, using their platform DokChain, a distributed network of transaction processors [157]. Change Healthcare, who are the leaders in blockchain technology in the healthcare sector, has recently been acquired, having implemented the first blockchain-based system at an enterprise scale to address claims management [158]. Table 2 summarizes the start-ups that have had an impact on Healthcare 4.0.

Table 2. Startups having an impact on Healthcare 4.0.

\begin{tabular}{cccc}
\hline References & Name & Technology & Methodology \\
\hline$[140]$ & anAPPhylaxis & BDA & Management of anaphylaxis \\
{$[141]$} & Egoo System & AI & Condition detection from biomarkers \\
{$[143]$} & Clarity & AI, ML & Early mental health condition detection \\
{$[146]$} & Feel & Pattern Recognition & Emotion-sensing mental condition detection \\
{$[148]$} & Bosetein & Deep Learning, Neural Network & Real-time optical food inspection \\
{$[149]$} & Fine Birth & AI & Recognizing Pre-term Labor (PTL) \\
{$[152]$} & Evitalz & Cloud Computing & Wireless smart devices for vital sign management \\
{$[154]$} & iSolve & blockchain & Secure drug supply chain \\
{$[156]$} & VAY Sports & AI, Computer Vision & Detection and tracking workout movements \\
{$[158]$} & PokitDok & blockchain & API development in large-scale enterprise \\
\hline
\end{tabular}

\section{Research Gaps and Challenges}

Having thoroughly reviewed the topics related to Industry 4.0, we identified room for improvement, as not every paper has covered the relevant topic sufficiently. In fact, we found gaps and challenges to be faced, which could be improved in the four following sectors.

\subsection{IoT and $B D A$}

IoT has brought about a significant revolution in the medical sector, and the healthcare industry has also benefited from it. The fourth industrial revolution has made it clear that the technologies used for development in every sector, including healthcare, are remarkable for purposes such as data storage and data analysis. Although the healthcare industry has gone through positive development with the assistance of IoT, there remain challenges; if these challenges are met, significant improvement can be made. Flexibility of performance and managing data volume is a critical challenge in IoT. Handling the diversity of scale also provides a challenge. If a hospital provides IoT services in devices using sensors, there should be proper diversity regarding the data. The data received by the sensors should be real, useful truths. If there is any damage to the device or the data is not accurate, the 
hospital or the healthcare organization may lose patients or staff. Another challenge is data exchange availability and resource privacy [159]. Sometimes, data needs to be transferred for different purposes; privacy is the main concern here, which should be ethically treated. Any incorrect information may cause a severe loss. Processing high-volume data requires adequate infrastructure. To read all the data that have originated from many devices, high network bandwidth is needed. As is well-known, IoT devices are generally connected with sensors. If the server is too big, it becomes difficult to obtain all of the data when using a slow network. Therefore, there is a need for high network bandwidth. It must also be considered that connected IoT devices could be at risk of hacking and leaking information unethically therefore, data transfer security should be ensured. Using a secondary network, unique and unguessable passwords, updating every device, and reducing cloud technology usage can prevent hacking. It may not be easy to maintain such groundwork, as IoT stores and transfers a huge amount of data [160]. There exist some technical problems, such as signal strength problems, as well as electromagnetic effects in health organizations and hospitals [161]. Ensuring health and safety is the primary objective of hospitals.

The main three challenges can be summarized into three categories: Security, trust, and loss of privacy. We discussed security in the beginning, and many researchers have been working on securing IoT devices and systems. No system can be $100 \%$ secure therefore, experts should identify and quantify possible security-related risks. Privacy is the main objective here, as patients expect to keep their information private or confidential. A hospital or medical center has the responsibility to ensure such confidentiality. As it is quite hard to manage and look after a huge portion of data, this is sometimes difficult. There exist laws regarding the protection of information in healthcare with the usage of IoT. In 1996, the USA passed the Health Insurance Probability and Accountability Act (HIPAA), which clearly emphasizes data protection [162]. The trust issue is also a challenge for IoT. Data transmission is enabled in IoT devices. During transmission, the data could be corrupted or diagnosed with malware. Unauthorized access could even occur. As this transferred information is used to make life-and-death decisions, if it is corrupted, then it may result in bad outcomes for patients. This type of problem is yet to be resolved. We know that caring is about making a person happy. The patients and sufferers always expect to get proper care and to be treated with trust. In the fourth industrial revolution, technology has reached the bedside of a patient. In most cases, nurses are not familiar with such use of IoT devices. Therefore, it is necessary to incorporate such devices while educating staff about how they are used to take care of the patient [163].

\subsection{Blockchain Technology}

The problems faced in implementing blockchain are problems faced by the whole healthcare sector regarding the privacy and safety of healthcare-related intelligence. The data stored on EHR systems are troublesome [126], and current systems in use are not entirely safe against disallowed entry or interchange of the data [21]. At present, the most popular EHR storage method is an architecture which is centralized and client-server based, gives those authoring the system full access to everything on the system. Such EHR storage creates a lack of privacy and leads to security flaws, whereby the secured data may be stolen, tampered with, or leaked. To combat these issues, security standards (e.g., HIPAA, DISHA, and COBIT) have been developed and implemented, in order to protect patient health information. Implementing blockchain-based EHR systems will allow the secure storage of healthcare information with the qualities that blockchain generally comprises, such as P2P networking, consensus protocol, and hash cryptography.

EHRs that are collected and stored in current systems are stored in such a way that they are non-portable, due to the various manners in which the data or information are recorded, making them non-interoperable. A commercial cause is that the institutions which collect data (EHR) do not generally feel devoted to making health records or data portable or approachable, considering the fear that making the data interoperable could lead to a loss of clients/patients, in the case that they decide to derive their services from a different insti- 
tution [106]. Healthcare institutions treat EHR data as their assets, creating inconveniences and high costs for patients when they need to transfer data to another healthcare supplier or provider. Several institutions have not responded correctly in adopting more advanced data management options worldwide, as they are required to accomplish the criteria of the Health Insurance Portability and Accountability Act (HIPAA) [164]. Regarding all of these aspects, blockchain technology provides a viable path for the diffusion and interoperability of medical information. EHR sharing with blockchain technology has some existing approaches [165]. A framework using the Ethereum blockchain has been proposed, called the Gem Health Network [166]. In Estonia, Guardtime, a company providing blockchain-based healthcare platforms, has established a system through which Estonian citizens, healthcare providers, and health insurance firms can obtain medical treatments [167]. There have also been proposals where, in order to reduce the exchange of large amounts of information through the system, a more reasonable method would be to only host metadata related to events, which can be health- and medical-related, on the blockchain [168,169]. In this case, the actual data or records are deposited in a global health cloud. There is no fully agreed-upon list of requirements that a blockchain-based medical data management system is required to possess, resulting in different methodologies having been proposed and used. Seven criteria which are essential for blockchain-based medical data management systems have been introduced in [96].

\section{3. $A I$}

The medical records, once transformed into digital records, are available for entry into a cloud server. Hackers or attackers can enter the cloud server, obtain unauthorized access, and easily violate an individual's personal and medical data. Therefore, challenges and struggles still remain. Ethical challenges come first. Some challenges are data confidentiality, privacy, and access control, as well as possession and administration. These are the fundamental problems and challenges, which can be categorized as ethical challenges. The main challenge here is to secure the complete image or access of the patient's data at a certain time. Users who have permission can see patient records from the EHR. Here, the problem is that an attacker or a hacker can steal the user's identity and check their information, which is risky and could be treated in the wrong way however, it is comparatively difficult to identify unauthorized access. For an unauthorized entry, confidentiality hides the critical information. Therefore, ensuring confidentiality is an essential task. Data accuracy is ensured through fidelity. Information can be modified by any attacker, which can lead to data inaccuracy. It is challenging to protect or prevent the stealing of data [124]. Hackers could possibly have the appropriate level of accessibility to obtain the ownership details and transform them into their ownership. They can disable access to the authorized entry. Policies should be highly maintained. For the safety of data involved in health diagnosis, security and strict administration are needed. To prevent theft, an EHR system should be developed in layers in other words, it should be developed with various indemnity patterns. Some websites allow online EHR systems with free limited storage space, which is risky. There is a possibility that they might sell the information, which can have some severe consequences for the patient or the staff. To make sure that the information is in the right hands is quite a challenging issue [170]. AI in Healthcare 4.0 can help to fight viruses however, as AI systems are still at a primordial stage, more time is needed before the results of such AI measures become visible [53]. In future, pandemics similar to COVID-19 will be easier to fight, through the use of Healthcare 4.0 technologies. Medical industries can adapt more futuristic technologies, such as AI, thus enabling the creation of a smarter healthcare infrastructure.

\subsection{Cloud Computing}

The usage of cloud computing has significantly increased in recent years. The fourth industrial revolution has had an incredible impact on the healthcare sector. Cloud computing has played a key role in contributing to this. To maintain proper cloud services, 
there exist many challenges. Although the adoption of cloud computing has been slow in medicine or healthcare sectors, the main challenges are security and interoperability [57]. Healthcare data should be strict, confidential, and private. If not, there is a high possibility that the data could be hacked or could be used in ways that are unethical. As cloud computing has made storing data more straightforward, it is necessary to make it more secure [171]. HIPAA compliance is the most practical and fundamental need while moving data to cloud storage [172]. HIPAA compliance is a way to update and maintain the data of patients. As a lot of data are stored, transferred, and updated regularly, there exists a high risk that these data can be hacked, leaked, and suffer from unauthorized access. HIPAA has been put in place to improve security. The HIPAA compliance checklist indicates a total of seven integral parts that are effective for compliant implementation [173]. The seven integrals are shown in Figure 10 [174].

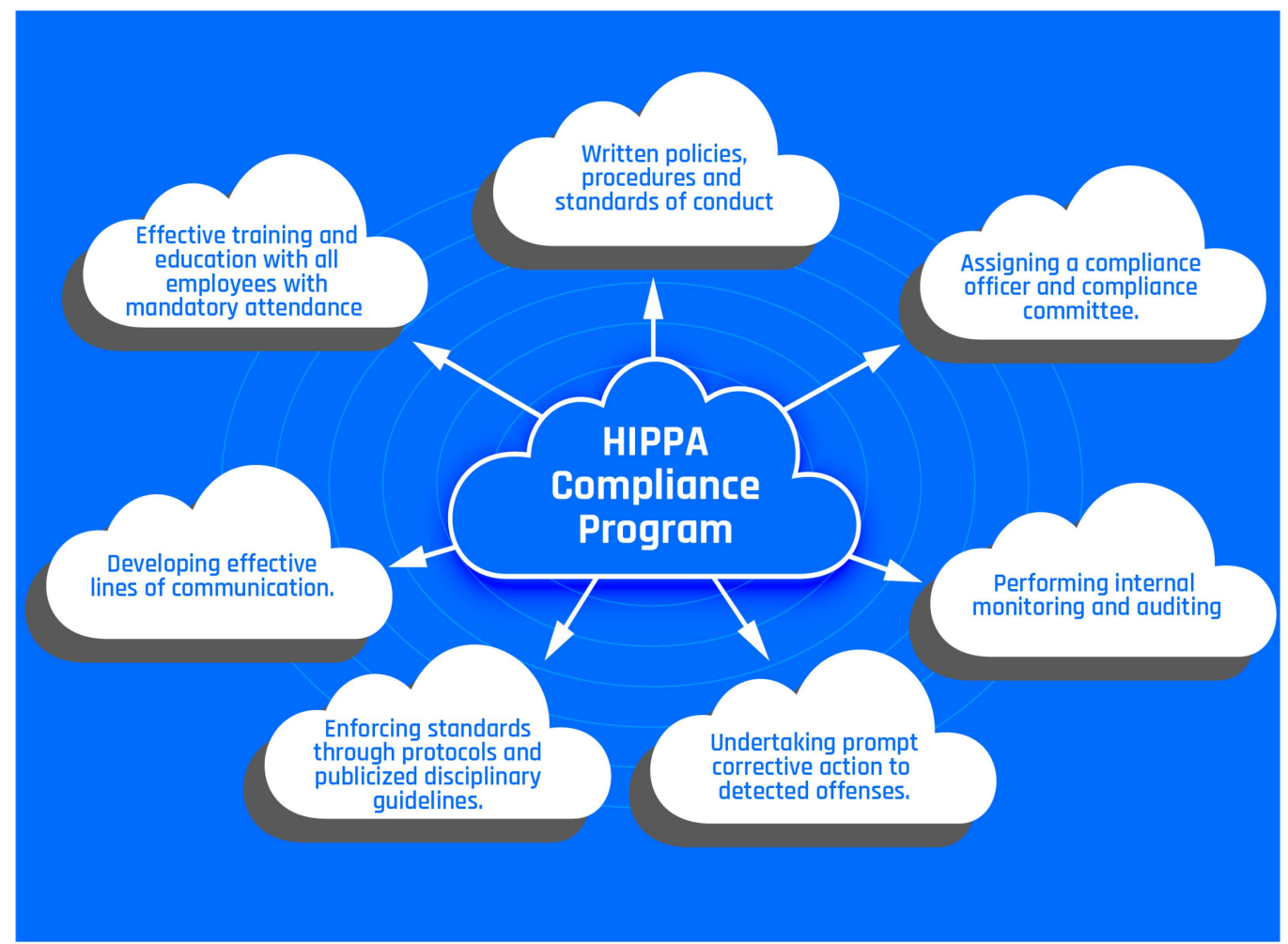

Figure 10. HIPAA compliance program [173].

Transferring a huge amount of data to a third-party is a challenging task. Healthcare data are necessary and sensitive and preventing unauthorized access, authentication, transmission security, and authorization must be ensured. The second factor is interoperabilitythe ability of a device or software to transfer and use information. Though cloud storage in healthcare is now in the fourth industrial revolution, healthcare system interoperability can occur in different ways, such as through the provider, software, computer, data levels, and transfer. Cloud providers generally maintain their independent data; to protect or strain the cloud, health organizations should complete their remaining procedures using cloud systems. A typical data model and framework should be used, in order to ensure interoperability [57]. Some interoperable systems are still in progress, as they have not yet been completed. Making current healthcare systems interoperable with cloud-based development could be difficult but, if it is done somehow, the healthcare sector may see many benefits [175]. 


\section{Conclusions}

The fourth industrial revolution had brought upon revolutionary ideas and changes in approaches for every industry, in a similar manner as previous industrial revolutions. The Industry 4.0 paradigm had given rise to new innovations and innovative approaches, enabling new ways to develop the healthcare sector. Various technologies, such as IoT, $\mathrm{BDA}$, blockchain, AI, and cloud computing, have enabled significant changes to take place and furthered the development of healthcare. By analyzing and surveying the relevant literature, we learned about and discussed the approaches and applications of these new methods, in terms of developing the industry. The present state of development and future challenges were discussed, along with their proposed solutions. The presented research gaps have also highlighted the shortcomings of the technology itself, as well as providing knowledge that can be used to improve that specific technology and enable it to become more usable for other purposes, not just in healthcare. Significant emphasis was put on blockchain technology, due to the benefits it could bring and its potential in multiple roles and industries. The section on start-ups (Section 3.5) was of particular importance, as no surveys of start-ups related to Healthcare 4.0, considering these technologies, were found in the existing literature. The start-ups section provided insight into the future of the healthcare industry, providing an overview of the ideas and approaches that are currently in use, as well as those that are soon to be used and implemented. With the advent of Industry 4.0 and the new technologies that have become available with it, the patterns of every industry is changing and new approaches are being explored. IoT, BDA, blockchain, $\mathrm{AI}$, and cloud computing are part of a group of technologies supporting the shift towards these futuristic approaches, as well as developments in every industry.

Funding: This research received no external funding.

Conflicts of Interest: The authors declare no conflict of interest.

\section{References}

1. Paul, S.; Rabbani, M.S.; Kundu, R.K.; Zaman, S.M.R. A review of smart technology (Smart Grid) and its features. In Proceedings of the 2014 1st International Conference on Non Conventional Energy (ICONCE 2014), Kalyani, India, 16-17 January 2014; pp. 200-203. [CrossRef]

2. Sunny, M.R.; Kabir, M.A.; Naheen, I.T.; Ahad, M.T. Residential Energy Management: A Machine Learning Perspective. In Proceedings of the 2020 IEEE Green Technologies Conference (GreenTech), Oklahoma City, OK, USA, 1-3 April 2020; pp. 229-234. [CrossRef]

3. Paul, S.; Ni, Z.; Mu, C. A Learning-Based Solution for an Adversarial Repeated Game in Cyber-Physical Power Systems. IEEE Trans. Neural Netw. Learn. Syst. 2020, 31, 4512-4523. [CrossRef]

4. Dengler, S.; Lahriri, S.; Trunzer, E.; Vogel-Heuser, B. Applied machine learning for a zero defect tolerance system in the automated assembly of pharmaceutical devices (DECSUP-D-20-00799R1). Decis. Support Syst. 2021, 146, 113540. [CrossRef]

5. Ni, Z.; Paul, S. A Multistage Game in Smart Grid Security: A Reinforcement Learning Solution. IEEE Trans. Neural Netw. Learn. Syst. 2019, 30, 2684-2695. [CrossRef] [PubMed]

6. Dal Mas, F.; Piccolo, D.; Cobianchi, L.; Edvinsson, L.; Presch, G.; Massaro, M.; Skrap, M.; Ferrario di Tor Vajana, A.; D’Auria, S.; Bagnoli, C. The effects of artificial intelligence, robotics, and industry 4.0 technologies. Insights from the Healthcare sector. In Proceedings of the First European Conference on the Impact of Artificial Intelligence and Robotics, Oxford, UK, 31 October-1 November 2019.

7. Boyes, H.; Hallaq, B.; Cunningham, J.; Watson, T. The industrial internet of things (IIoT): An analysis framework. Comput. Ind. 2018, 101, 1-12. [CrossRef]

8. Lasi, H.; Fettke, P.; Kemper, H.G.; Feld, T.; Hoffmann, M. Industry 4.0. Bus. Inf. Syst. Eng. 2014, 6, 239-242. [CrossRef]

9. Ashton, T.S. The Industrial Revolution 1760-1830; Praeger: Westport, CT, USA, 1997.

10. Philbeck, T.; Davis, N. The fourth industrial revolution. J. Int. Aff. 2018, 72, 17-22.

11. Aceto, G.; Persico, V.; Pescapé, A. Industry 4.0 and health: Internet of things, big data, and cloud computing for healthcare 4.0. J. Ind. Inf. Integr. 2020, 18, 100129. [CrossRef]

12. Liao, Y.; Deschamps, F.; Loures, E.d.F.R.; Ramos, L.F.P. Past, present and future of Industry 4.0-A systematic literature review and research agenda proposal. Int. J. Prod. Res. 2017, 55, 3609-3629. [CrossRef]

13. Kamble, S.S.; Gunasekaran, A.; Gawankar, S.A. Sustainable Industry 4.0 framework: A systematic literature review identifying the current trends and future perspectives. Process. Saf. Environ. Prot. 2018, 117, 408-425. [CrossRef] 
14. Jayaraman, P.P.; Forkan, A.R.M.; Morshed, A.; Haghighi, P.D.; Kang, Y.B. Healthcare 4.0: A review of frontiers in digital health. Wiley Interdiscip. Rev. Data Min. Knowl. Discov. 2020, 10, e1350. [CrossRef]

15. Tortorella, G.L.; Fogliatto, F.S.; Mac Cawley Vergara, A.; Vassolo, R.; Sawhney, R. Healthcare 4.0: Trends, challenges and research directions. Prod. Plan. Control. 2019, 31, 1245-1260. [CrossRef]

16. Kumari, A.; Tanwar, S.; Tyagi, S.; Kumar, N. Fog computing for Healthcare 4.0 environment: Opportunities and challenges Comput. Electr. Eng. 2018, 72, 1-13. [CrossRef]

17. Islam, S.R.; Kwak, D.; Kabir, M.H.; Hossain, M.; Kwak, K.S. The internet of things for health care: A comprehensive survey. IEEE Access 2015, 3, 678-708. [CrossRef]

18. Mahmud, M.; Kaiser, M.S.; Rahman, M.M.; Rahman, M.A.; Shabut, A.; Al-Mamun, S.; Hussain, A. A brain-inspired trust management model to assure security in a cloud based IoT framework for neuroscience applications. Cogn. Comput. 2018, 10, 864-873. [CrossRef]

19. Solution Blockchain de Traçabilité des Médicaments. 2020. Available online: https:/ /www.blockpharma.com/ (accessed on 21 September 2020).

20. What Does Connecting Care Mean for People in Bristol, North Somerset and South Gloucestershire? 2020. Available online: https: / / www.connectingcarebnssg.co.uk/ (accessed on 21 September 2020).

21. Chen, L.; Lee, W.K.; Chang, C.C.; Choo, K.K.R.; Zhang, N. Blockchain based searchable encryption for electronic health record sharing. Future Gener. Comput. Syst. 2019, 95, 420-429. [CrossRef]

22. Li, H.; Zhu, L.; Shen, M.; Gao, F.; Tao, X.; Liu, S. Blockchain-based data preservation system for medical data. J. Med. Syst. 2018, 42, 141. [CrossRef]

23. Ethereum. 2020. Available online: https:/ / ethereum.org/en/ (accessed on 21 September 2020).

24. Fan, K.; Wang, S.; Ren, Y.; Li, H.; Yang, Y. Medblock: Efficient and secure medical data sharing via blockchain. J. Med. Syst. 2018, 42, 136. [CrossRef]

25. Guo, R.; Shi, H.; Zhao, Q.; Zheng, D. Secure attribute-based signature scheme with multiple authorities for blockchain in electronic health records systems. IEEE Access 2018, 6, 11676-11686. [CrossRef]

26. Sun, Y.; Zhang, R.; Wang, X.; Gao, K.; Liu, L. A decentralizing attribute-based signature for healthcare blockchain. In Proceedings of the 2018 27th International Conference on Computer Communication and Networks (ICCCN), Hangzhou, China, 30 July-2 August 2018; pp. 1-9.

27. Yang, G.; Li, C. A design of blockchain-based architecture for the security of electronic health record (EHR) systems. In Proceedings of the 2018 IEEE International Conference on Cloud Computing Technology and Science (CloudCom), Nicosia, Cyprus, 10-13 December 2018; pp. 261-265.

28. SimplyVital Health. 2020. Available online: https://www.f6s.com/simplyvitalhealth/ (accessed on 21 September 2020).

29. SimplyVital Health. SimplyVital Health and Lacuna Health Announce Strategic Partnership to Drive Savings for Physicians in Value-Based Care Programs. 2020. Available online: https://www.prnewswire.com/news-releases/simplyvital-healthand-lacuna-health-announce-strategic-partnership-to-drive-savings-for-physicians-in-value-based-care-programs-300983910. html (accessed on 21 September 2020).

30. Medicalchain. 2020. Available online: https://medicalchain.com/en/ (accessed on 21 September 2020).

31. Liu, W.; Zhu, S.; Mundie, T.; Krieger, U. Advanced block-chain architecture for e-health systems. In Proceedings of the 2017 IEEE 19th International Conference on e-Health Networking, Applications and Services (Healthcom), Dalian, China, 12-15 October 2017; pp. 1-6.

32. Azaria, A.; Ekblaw, A.; Vieira, T.; Lippman, A. Medrec: Using blockchain for medical data access and permission management. In Proceedings of the 2016 2nd International Conference on Open and Big Data (OBD), Vienna, Austria, 22-24 August 2016; pp. 25-30.

33. MedRec. 2020. Available online: https://medrec.media.mit.edu/ (accessed on 21 September 2020).

34. Zhang, J.; Xue, N.; Huang, X. A secure system for pervasive social network-based healthcare. IEEE Access 2016, 4, 9239-9250. [CrossRef]

35. Xia, Q.; Sifah, E.B.; Asamoah, K.O.; Gao, J.; Du, X.; Guizani, M. MeDShare: Trust-less medical data sharing among cloud service providers via blockchain. IEEE Access 2017, 5, 14757-14767. [CrossRef]

36. Jiang, S.; Cao, J.; Wu, H.; Yang, Y.; Ma, M.; He, J. Blochie: A blockchain-based platform for healthcare information exchange. In Proceedings of the 2018 IEEE International Conference on Smart Computing (Smartcomp), Sicily, Italy, 18-20 June 2018; pp. 49-56.

37. Wang, H.; Song, Y. Secure cloud-based EHR system using attribute-based cryptosystem and blockchain. J. Med. Syst. 2018, 42, 152. [CrossRef] [PubMed]

38. Patientory-Healthcare Platform for Healthcare Providers and Consumers. 2020. Available online: https://patientory.com/ (accessed on 21 September 2020).

39. Yue, X.; Wang, H.; Jin, D.; Li, M.; Jiang, W. Healthcare data gateways: Found healthcare intelligence on blockchain with novel privacy risk control. J. Med. Syst. 2016, 40, 218. [CrossRef] [PubMed]

40. Zhang, X.; Poslad, S. blockchain support for flexible queries with granular access control to electronic medical records (EMR). In Proceedings of the 2018 IEEE International Conference on Communications (ICC), Kansas City, MO, USA, 20-24 May 2018; pp. 1-6. 
41. Koepsell, D. The DNA Data Marketplace. 2020. Available online: https://encrypgen.com/ (accessed on 21 September 2020).

42. Liang, X.; Zhao, J.; Shetty, S.; Liu, J.; Li, D. Integrating blockchain for data sharing and collaboration in mobile healthcare applications. In Proceedings of the 2017 IEEE 28th Annual International Symposium on Personal, Indoor, and Mobile Radio Communications (PIMRC), Montreal, QC, Canada, 8-13 October 2017; pp. 1-5.

43. MyCoralHealth. 2020. Available online: https://mycoralhealth.com/ (accessed on 21 September 2020).

44. Batista, M. Medgadget. 2018. Available online: https://www.medgadget.com/2018/08/healthcare-blockchain-startup-coralhealth-announces-health-records-app-and-upcoming-token-sale-interview.html (accessed on 21 September 2020).

45. Chronicled. 2020. Available online: https:/ / chronicled.com/ (accessed on 21 September 2020).

46. Thakkar, P.; Nathan, S.; Viswanathan, B. Performance benchmarking and optimizing hyperledger fabric blockchain platform. In Proceedings of the 2018 IEEE 26th International Symposium on Modeling, Analysis, and Simulation of Computer and Telecommunication Systems (MASCOTS), Milwaukee, WI, USA, 25-28 September 2018; pp. 264-276.

47. Hyperledger Fabric. 2020. Available online: https://www.hyperledger.org/use/fabric (accessed on 21 September 2020).

48. Sukhwani, H.; Martínez, J.M.; Chang, X.; Trivedi, K.S.; Rindos, A. Performance modeling of PBFT consensus process for permissioned blockchain network (hyperledger fabric). In Proceedings of the 2017 IEEE 36th Symposium on Reliable Distributed Systems (SRDS), Hong Kong, China, 26-29 September 2017; pp. 253-255.

49. Cyran, M.A. Blockchain as a foundation for sharing healthcare data. Blockchain Healthc. Today 2018, 1, 1-6. [CrossRef]

50. Gorenflo, C.; Lee, S.; Golab, L.; Keshav, S. Fastfabric: Scaling hyperledger fabric to 20,000 transactions per second. In Proceedings of the 2019 IEEE International Conference on Blockchain and Cryptocurrency (ICBC), Seoul, Korea, 14-17 May 2019 ; pp. 455-463.

51. Uddin, M.A.; Stranieri, A.; Gondal, I.; Balasubramanian, V. Continuous patient monitoring with a patient centric agent: A block architecture. IEEE Access 2018, 6, 32700-32726. [CrossRef]

52. Kuo, T.T.; Ohno-Machado, L. Modelchain: Decentralized privacy-preserving healthcare predictive modeling framework on private blockchain networks. arXiv 2018, arXiv:1802.01746.

53. Petropoulos, G. Artificial Intelligence in the Fight against COVID-19. 2020. Available online: https://www.bruegel.org/2020/03/ artificial-intelligence-in-the-fight-against-covid-19/ (accessed on 9 October 2020).

54. Haleem, A.; Javaid, M.; Vaishya, R.; Deshmukh, S. Areas of academic research with the impact of COVID-19. Am. J. Emerg. Med. 2020, 38, 1524-1526. [CrossRef]

55. Javaid, M.; Haleem, A.; Vaishya, R.; Bahl, S.; Suman, R.; Vaish, A. Industry 4.0 technologies and their applications in fighting COVID-19 pandemic. Diabetes Metab. Syndr. Clin. Res. Rev. 2020, 14, 419-422. [CrossRef]

56. Harris, J.G.; Alter, A.E. Six questions every Executive should ask about cloud computing. Accent. Inst. High Perform. 2010.

57. Ahuja, S.P.; Mani, S.; Zambrano, J. A survey of the state of cloud computing in healthcare. Netw. Commun. Technol. 2012, 1, 12. [CrossRef]

58. Zhang, R.; Liu, L. Security models and requirements for healthcare application clouds. In Proceedings of the 2010 IEEE 3rd International Conference on cloud Computing, Miami, FL, USA, 5-10 July 2010; pp. 268-275.

59. Kuo, M.H. Opportunities and challenges of cloud computing to improve health care services. J. Med. Internet Res. 2011, 13, e67. [CrossRef]

60. Mohajan, H. The First Industrial Revolution: Creation of a New Global Human Era. J. Soc. Sci. Humanit. $2019,5,377-387$.

61. Engelman, R.; Stern, A.E.; Silva, J.O. U.S. History Scene. The Second Industrial Revolution. US History Scene. Available online: https: / / ushistoryscene.com/article/second-industrial-revolution/ (accessed on 1 June 2021).

62. Pouspourika, K. The 4 Industrial Revolutions. 2019. Available online: https://ied.eu/project-updates/the-4-industrialrevolutions / (accessed on 9 October 2020).

63. I-SCOOP. Industry 4.0: The Fourth Industrial Revolution-Guide to Industrie 4.0. 2017. Available online: https: / / www.i-scoop. eu/industry-4-0/ (accessed on 9 October 2020).

64. Tay, S.I.; Lee, T.C.; Hamid, N.Z.A.; Ahmad, A.N.A. An overview of industry 4.0: Definition, components, and government initiatives. J. Adv. Res. Dyn. Control. Syst. 2018, 10, 1379-1387.

65. Schwab, K. The Fourth Industrial Revolution: What It Means, How to Respond; World Economic Forum; Crown Business: New York, NY, USA, 2016; Volume 14, p. 2016.

66. Time to Join the Digital Dots. 2018. Available online: https://www.aero-mag.com/meggitt-applied-research-technology-groupdata-capture/ (accessed on 9 October 2020).

67. White Paper Work 4.0; Federal Ministry of Labour and Social Affairs. 2016. Available online: https://www.bmas.de/EN/ Services/Publications / a883-white-paper.html (accessed on 9 October 2020).

68. Hermann, M.; Pentek, T.; Otto, B. Design principles for industrie 4.0 scenarios. In Proceedings of the 201649 th Hawaii International Conference on System Sciences (HICSS), Koloa, HI, USA, 5-8 January 2016; pp. 3928-3937.

69. Bonner, M. What Is Industry 4.0 and What Does It Mean for My Manufacturing? Saint Claire Syst. 2017. Available online: https: / /blog.viscosity.com/blog/what-is-industry-4.0-and-what-does-it-mean-for-mymanufacturing (accessed on 29 December 2018).

70. Marr, B. What Everyone Must Know about Industry 4.0; Forbes Tech: Jersey City, NJ, USA, 2016.

71. Gronau, N.; Grum, M.; Bender, B. Determining the optimal level of autonomy in cyber-physical production systems. In Proceedings of the 2016 IEEE 14th International Conference on Industrial Informatics (INDIN), Poitiers, France, 18-21 July 2016; pp. 1293-1299. 
72. Hartog, T.; Marshall, M.; Alhashim, A.G.; Ahad, M.T.; Siddique, Z. Work in Progress: Using Neuro-responses to Understand Creativity, the Engineering Design Process, and Concept Generation. In Proceedings of the 2020 ASEE Virtual Annual Conference Content Access, Virtual Online. 22-26 June 2020; Available online: https:/ / peer.asee.org/35701 (accessed on 1 June 2021).

73. Hartog, T.; Marshall, M.; Ahad, M.T.; Alhashim, A.G.; Kremer, G.O.; van Hell, J.; Siddique, Z. Pilot Study: Investigating EEG Based Neuro-Responses of Engineers via a Modified Alternative Uses Task to Understand Creativity. In Proceedings of the International Design Engineering Technical Conferences and Computers and Information in Engineering Conference, 2020; Volume 3: 17th International Conference on Design Education (DEC), V003T03A019, Online. 17-19 August 2020. [CrossRef]

74. Ahad, M.T.; Rahman, A. An Awareness Study of Smart Meters Radiation on Human Head. In Proceedings of the 2020 IEEE Green Technologies Conference(GreenTech), Oklahoma City, OK, USA, 1-3 April 2020; pp. 223-228. [CrossRef]

75. Gottge, S.; Menzel, T.; Forslund, H. Industry 4.0 technologies in the purchasing process. Ind. Manag. Data Syst. 2020, 120, 730-748. [CrossRef]

76. Javaid, M.; Haleem, A. Industry 4.0 applications in medical field: A brief review. Curr. Med. Res. Pract. 2019, 9, 102-109. [CrossRef]

77. Yazdan, M.M.S.; Ahad, M.T.; Jahan, I.; Mazumder, M. Review on the Evaluation of the Impacts of Wastewater Disposal in Hydraulic Fracturing Industry in the United States. Technologies 2020, 8, 67. [CrossRef]

78. Zhang, W.; Ma, F.; Ren, M.; Yang, F. Application with Internet of things technology in the municipal industrial wastewater treatment based on membrane bioreactor process. Appl. Water Sci. 2021, 11, 1-12. [CrossRef]

79. Al Hossain, B.M.T.; Ahmed, T.; Aktar, M.N.; Fida, M.; Khan, A.; Islam, A.S.; Yazdan, M.M.S.; Noor, F.; Rahaman, A.Z. Climate Change Impacts on Water Availability in the Meghna Basin. In Proceedings of the 5th International Conference on Water and Flood Management (ICWFM-2015), Dhaka, Bangladesh, 6-8 March 2015.

80. Singh, R. Re-Envisioning Remote Sensing Applications: Perspectives from Developing Countries; CRC Press: Boca Raton, FL, USA, 2021.

81. Shafi, U.; Mumtaz, R.; Iqbal, N.; Zaidi, S.M.H.; Zaidi, S.A.R.; Hussain, I.; Mahmood, Z. A Multi-Modal Approach for Crop Health Mapping Using Low Altitude Remote Sensing, Internet of Things (IoT) and Machine Learning. IEEE Access 2020, 8 , 112708-112724. [CrossRef]

82. Yazdan, M.M.S.; Rahaman, A.Z.; Noor, F.; Duti, B.M. Establishment of co-relation between remote sensing based trmm data and ground based precipitation data in north-east region of bangladesh. In Proceedings of the 2nd International Conference on Civil Engineering for Sustainable Development (ICCESD-2014), KUET, Khulna, Bangladesh, 14-16 February 2014; pp. 14-16.

83. Sunburn Sensor Among Top Techs at Nanomedicine Conference. Available online: https://newsroom.unsw.edu.au/news/ health/sunburn-sensor-among-top-techs-nanomedicine-conference\#: \{\}:text=Chemists (accessed on 1 June 2021).

84. Lu, Y.; Papagiannidis, S.; Alamanos, E. Internet of Things: A systematic review of the business literature from the user and organisational perspectives. Technol. Forecast. Soc. Chang. 2018, 136, 285-297. [CrossRef]

85. Estrela, V.V.; Monteiro, A.C.B.; França, R.P.; Iano, Y.; Khelassi, A.; Razmjooy, N. Health 4.0: Applications, management, technologies and review. Med. Technol. J. 2018, 2, 262-276.

86. Durga, S.; Nag, R.; Daniel, E. Survey on machine learning and deep learning algorithms used in internet of things (IoT) healthcare. In Proceedings of the 2019 3rd International Conference on Computing Methodologies and Communication (ICCMC), Erode, India, 27-29 March 2019; pp. 1018-1022.

87. Matin, M.A.; Islam, M. Overview of wireless sensor network. In Wireless Sensor Networks-Technology and Protocols; INTECH: London, UK, 2012; pp. 1-3.

88. Aloi, G.; Fortino, G.; Gravina, R.; Pace, P.; Savaglio, C. Simulation-driven platform for Edge-based AAL systems. IEEE J. Sel. Areas Commun. 2020, 39, 446-462. [CrossRef]

89. Alloghani, M.; Al-Jumeily, D.; Hussain, A.; Aljaaf, A.J.; Mustafina, J.; Petrov, E. Healthcare services innovations based on the state of the art technology trend industry 4.0. In Proceedings of the 2018 11th International Conference on Developments in eSystems Engineering (DeSE), Cambridge, UK, 2-5 September 2018; pp. 64-70.

90. Lee, E.A. Cyber physical systems: Design Challenges. In Proceedings of the 11th IEEE International Symposium on Object and Component-Oriented Real-Time Distributed Computing (ISORC), Orlando, FL, USA, 5-7 May 2008.

91. Dimitrov, D.V. Medical internet of things and big data in healthcare. Healthc. Inform. Res. 2016, 22, 156-163. [CrossRef] [PubMed]

92. Zawadzki, P.; Żywicki, K. Smart product design and production control for effective mass customization in the Industry 4.0 concept. Manag. Prod. Eng. Rev. 2016, 7, 105-112. [CrossRef]

93. Wiesner, M.; Pfeifer, D. Health recommender systems: Concepts, requirements, technical basics and challenges. Int. J. Environ. Res. Public Health 2014, 11, 2580-2607. [CrossRef]

94. Manogaran, G.; Thota, C.; Lopez, D.; Sundarasekar, R. Big data security intelligence for healthcare industry 4.0. In Cybersecurity for Industry 4.0; Springer: Berlin/Heisenberg, Germany, 2017; pp. 103-126.

95. Kuo, T.T.; Kim, H.E.; Ohno-Machado, L. Blockchain distributed ledger technologies for biomedical and health care applications. J. Am. Med. Inform. Assoc. 2017, 24, 1211-1220. [CrossRef]

96. Zhang, P.; Walker, M.A.; White, J.; Schmidt, D.C.; Lenz, G. Metrics for assessing blockchain-based healthcare decentralized apps. In Proceedings of the 2017 IEEE 19th International Conference on e-Health Networking, Applications and Services (Healthcom), Dalian, China, 12-15 October 2017; pp. 1-4.

97. Faramondi, L.; Oliva, G.; Setola, R.; Vollero, L. Iiot in the hospital scenario: Hospital 4.0, blockchain and robust data management. In Security and Privacy Trends in the Industrial Internet of Things; Springer: Berlin/Heisenberg, Germany, 2019; pp. 271-285. 
98. Vora, J.; Nayyar, A.; Tanwar, S.; Tyagi, S.; Kumar, N.; Obaidat, M.S.; Rodrigues, J.J. BHEEM: A blockchain-based framework for securing electronic health records. In Proceedings of the 2018 IEEE Globecom Workshops (GC Wkshps), Abu Dhabi, United Arab Emirates, 9-13 December 2018; pp. 1-6.

99. Tanwar, S.; Parekh, K.; Evans, R. Blockchain-based electronic healthcare record system for healthcare 4.0 applications. J. Inf. Secur. Appl. 2020, 50, 102407. [CrossRef]

100. Clauson, K.A.; Breeden, E.A.; Davidson, C.; Mackey, T.K. Leveraging blockchain technology to enhance supply chain management in healthcare: An exploration of challenges and opportunities in the health supply chain. Blockchain Healthc. Today 2018, 1, 1-12.

101. Dagher, G.G.; Mohler, J.; Milojkovic, M.; Marella, P.B. Ancile: Privacy-preserving framework for access control and interoperability of electronic health records using blockchain technology. Sustain. Cities Soc. 2018, 39, 283-297. [CrossRef]

102. Rifi, N.; Rachkidi, E.; Agoulmine, N.; Taher, N.C. Towards using blockchain technology for eHealth data access management. In Proceedings of the 2017 Fourth International Conference on Advances in Biomedical Engineering (ICABME), Hadat-Beirut, Lebanon, 19-21 October 2017; pp. 1-4.

103. Thuemmler, C.; Bai, C. Health 4.0: Application of industry 4.0 design principles in future asthma management. In Health 4.0: How Virtualization and Big Data Are Revolutionizing Healthcare; Springer: Berlin/Heisenberg, Germany, 2017; pp. $23-37$.

104. Wainer, J.; Campos, C.; Salinas, M.; Sigulem, D. Security requirements for a lifelong electronic health record system: An opinion. Open Med. Inform. J. 2008, 2, 160. [CrossRef]

105. Gunter, T.D.; Terry, N.P. The emergence of national electronic health record architectures in the United States and Australia: Models, costs, and questions. J. Med. Internet Res. 2005, 7, e3. [CrossRef] [PubMed]

106. Ivan, D. Moving toward a blockchain-based method for the secure storage of patient records. In ONC/NIST Use of Blockchain for Healthcare and Research Workshop; ONC/NIST: Gaithersburg, MD, USA, 2016; pp. 1-11.

107. Carey, P. Data Protection: A Practical Guide to UK and EU Law; Oxford University Press, Inc.: Oxford, UK, 2018.

108. Paul, S.; Ding, F.; Kumar, U.; Liu, W.; Ni, Z. Q-Learning-Based Impact Assessment of Propagating Extreme Weather on Distribution Grids. In Proceedings of the 2020 IEEE Power Energy Society General Meeting (PESGM), Montreal, QC, Canada, 2-6 August 2020; pp. 1-5. [CrossRef]

109. Thomas, S.; Palahnuk, H.; Amini, H.; Akseli, I. Data-smart machine learning methods for predicting composition-dependent Young's modulus of pharmaceutical compacts. Int. J. Pharm. 2021, 592, 120049. [CrossRef]

110. Luan, H.; Tsai, C.C. A review of using machine learning approaches for precision education. Educ. Technol. Soc. 2021, 24, 250-266.

111. Paul, S.; Ni, Z.; Ding, F. An Analysis of Post Attack Impacts and Effects of Learning Parameters on Vulnerability Assessment of Power Grid. In Proceedings of the 2020 IEEE Power Energy Society Innovative Smart Grid Technologies Conference (ISGT), Washington, DC, USA, 17-20 February 2020; pp. 1-5. [CrossRef]

112. Yousuf, H.; Zainal, A.Y.; Alshurideh, M.; Salloum, S.A. Artificial intelligence models in power system analysis. In Artificial Intelligence for Sustainable Development: Theory, Practice and Future Applications; Springer: Berlin/Heisenberg, Germany, 2021; pp. 231-242.

113. Paul, S.; Ding, F. Identification of Worst Impact Zones for Power Grids During Extreme Weather Events Using Q-learning. In Proceedings of the 2020 IEEE Power Energy Society Innovative Smart Grid Technologies Conference (ISGT), Washington, DC, USA, 17-20 February 2020; pp. 1-5. [CrossRef]

114. Devarapalli, R.; Bhattacharyya, B.; Sinha, N.K.; Dey, B. Amended gwo approach based multi-machine power system stability enhancement. ISA Trans. 2021, 109, 152-174. [CrossRef] [PubMed]

115. Paul, S.; Haq, M.R.; Das, A.; Ni, Z. A Comparative Study of Smart Grid Security Based on Unsupervised Learning and Load Ranking. In Proceedings of the 2019 IEEE International Conference on Electro Information Technology (EIT), Brookings, SD, USA, 20-22 May 2019; pp. 310-315. [CrossRef]

116. Udgata, S.K.; Suryadevara, N.K. COVID-19, Sensors, and Internet of Medical Things (IoMT). In Internet of Things and Sensor Network for COVID-19; Springer: Singapore, 2021; pp. 39-53.

117. Haleem, A.; Javaid, M. Additive manufacturing applications in industry 4.0: A review. J. Ind. Integr. Manag. $2019,4,1930001$. [CrossRef]

118. Riffat, M.; Yasir, A.; Naheen, I.T.; Paul, S.; Ahad, M.T. Augmented Reality for Smarter Bangladesh. In Proceedings of the 2020 IEEE Green Technologies Conference(GreenTech), Oklahoma City, OK, USA, 1-3 April 2020; pp. 217-222. [CrossRef]

119. Ren, J.l.; Zhang, A.H.; Wang, X.J. Traditional Chinese medicine for COVID-19 treatment. Pharmacol. Res. 2020, 155, 104743. [CrossRef] [PubMed]

120. Ahsan, M.M.; Ahad, M.T.; Soma, F.A.; Paul, S.; Chowdhury, A.; Luna, S.A.; Yazdan, M.M.S.; Rahman, A.; Siddique, Z.; Huebner, P. Detecting SARS-CoV-2 From Chest X-Ray Using Artificial Intelligence. IEEE Access 2021, 9, 35501-35513. [CrossRef]

121. Ruan, Q.; Yang, K.; Wang, W.; Jiang, L.; Song, J. Clinical predictors of mortality due to COVID-19 based on an analysis of data of 150 patients from Wuhan, China. Intensive Care Med. 2020, 46, 846-848. [CrossRef]

122. Ienca, M.; Vayena, E. On the responsible use of digital data to tackle the COVID-19 pandemic. Nat. Med. 2020, 26, 463-464. [CrossRef]

123. Hassan, N.H.; Ismail, Z. A conceptual model for investigating factors influencing information security culture in healthcare environment. Procedia Soc. Behav. Sci. 2012, 65, 1007-1012. [CrossRef]

124. Hathaliya, J.J.; Tanwar, S. An exhaustive survey on security and privacy issues in Healthcare 4.0. Comput. Commun. 2020, 153, 311-335. [CrossRef] 
125. He, D.; Zeadally, S. Authentication protocol for an ambient assisted living system. IEEE Commun. Mag. 2015, 53, 71-77. [CrossRef]

126. Hathaliya, J.J.; Tanwar, S.; Tyagi, S.; Kumar, N. Securing electronics healthcare records in healthcare 4.0: A biometric-based approach. Comput. Electr. Eng. 2019, 76, 398-410. [CrossRef]

127. Hasin, Y.; Seldin, M.; Lusis, A. Multi-omics approaches to disease. Genome Biol. 2017, 18, 1-15. [CrossRef]

128. Wang, F.; Preininger, A. AI in health: State of the art, challenges, and future directions. Yearb. Med. Inform. 2019, 28, 16. [CrossRef]

129. Fridley, B.L.; Lund, S.; Jenkins, G.D.; Wang, L. AB ayesian Integrative Genomic Model for Pathway Analysis of Complex Traits. Genet. Epidemiol. 2012, 36, 352-359. [CrossRef]

130. Baytas, I.M.; Xiao, C.; Wang, F.; Jain, A.K.; Zhou, J. Heterogeneous hyper-network embedding. In Proceedings of the 2018 IEEE International Conference on Data Mining (ICDM), Singapore, 17-20 November 2018; pp. 875-880.

131. Jensen, P.B.; Jensen, L.J.; Brunak, S. Mining electronic health records: Towards better research applications and clinical care. Nat. Rev. Genet. 2012, 13, 395-405. [CrossRef] [PubMed]

132. Mikolov, T.; Karafiát, M.; Burget, L.Č.; Černockỳ, J.J.; Khudanpur, S. Recurrent neural network based language model. In Proceedings of the 11th Annual Conference of the International Speech Communication Association (INTERSPEECH-2010), Makuhari, Japan, 26-30 September 2010.

133. Wu, J.; Roy, J.; Stewart, W.F. Prediction modeling using EHR data: Challenges, strategies, and a comparison of machine learning approaches. Med. Care 2010, 48, S106-S113. [CrossRef]

134. Choi, E.; Schuetz, A.; Stewart, W.F.; Sun, J. Using recurrent neural network models for early detection of heart failure onset. J. Am. Med. Inform. Assoc. 2017, 24, 361-370. [CrossRef] [PubMed]

135. Hannun, A.Y.; Rajpurkar, P.; Haghpanahi, M.; Tison, G.H.; Bourn, C.; Turakhia, M.P.; Ng, A.Y. Cardiologist-level arrhythmia detection and classification in ambulatory electrocardiograms using a deep neural network. Nat. Med. 2019, 25, 65. [CrossRef] [PubMed]

136. Schwab, P.; Scebba, G.C.; Zhang, J.; Delai, M.; Karlen, W. Beat by beat: Classifying cardiac arrhythmias with recurrent neural networks. In Proceedings of the 2017 Computing in Cardiology (CinC), Rennes, France, 24-27 September 2017; pp. 1-4.

137. Tang, F.; Lin, K.; Uchendu, I.; Dodge, H.H.; Zhou, J. Improving mild cognitive impairment prediction via reinforcement learning and dialogue simulation. arXiv 2018, arXiv:1802.06428.

138. Garets, D.; Davis, M. Electronic medical records vs. electronic health records: Yes, there is a difference. In Policy White Paper; HIMSS Analytics: Chicago, IL, USA, 2006; pp. 1-14.

139. Adan Medical Innovation. 2020. Available online: https:/ /www.adanmi.com/ (accessed on 9 October 2020).

140. Martin, J. AnAPPhylaxis: Building a Safe, Clean Medical App for a Life-Threatening Condition. 2019. Available online: https://mobilejazz.com/blog/anapphylaxis-building-a-safe-clean-medical-app-for-a-life-threatening-condition/ (accessed on 9 October 2020).

141. Smith, J. This Startup Is Developing a DIY Biomarker Diagnostics Kit. 2020. Available online: https://www.labiotech. eu/diagnostics / qlife-diagnostics-diy /\#: \{\}:text=The \%20company \T1 \textquoterights \%20technology $\% 20 \% \mathrm{E} 2 \% 80 \% 94 \% 2$ 0called $\% 20$ the, by $\% 20$ the $\% 20$ type $\% 20$ of $\% 20$ cartridge (accessed on 9 October 2020).

142. How Healthy Are You? 2020. Available online: https://www.egoo.health/ (accessed on 9 October 2020).

143. Clarigent Health Launches Clairity, Tracking Vocal Biomarkers To Support Mental Health Risk Reduction. 2020. Available online: https: / / aithority.com/technology/life-sciences/clarigent-health-launches-clairity/ (accessed on 9 October 2020).

144. Clairity Launch. 2020. Available online: https://clarigenthealth.com/our-products-1/ (accessed on 9 October 2020).

145. Feel Program. 2020. Available online: https://www.myfeel.co/feel-program/ (accessed on 9 October 2020).

146. Kryl, C. In the COVID-19 Era, This Mental Health Startup Wants Users to Feel Relief. 2020. Available online: https://matter. health/posts / covid-19-startup-feature-sentio-solutions / (accessed on 9 October 2020).

147. We Ensure the Safety of Your Food. Available online: https://bosetein.com/ (accessed on 9 October 2020).

148. How Do you Know if your Food Is Still Good to Eat? 2020. Available online: https://www.de-hub.de/en/blog/d/how-do-youknow-if-your-food-is-still-good-to-eat/ (accessed on 9 October 2020).

149. Hinchliffe, T. Fundación Botín to Invest $844 \mathrm{~K}$ in Spanish Biotech Startups. Innitius, EpiDisease. 2018. Available online: https://novobrief.com/spanish-biotech-startups/6945/ (accessed on 9 October 2020).

150. INNITIUS: Developing and Commercializing Novel Devices to Improve Patient Outcomes and Lower the Costs of Preterm Labor. 2020. Available online: https:/ / www.innitius.com/ (accessed on 9 October 2020).

151. Evitalz Telehealth Kit. 2020. Available online: https:/ /www.evitalz.com/telehealth-kit.html/ (accessed on 9 October 2020).

152. Updates from the ISfTeH Global Telemedicine \& eHealth Network (April 2019). 2019. Available online: https://myemail. constantcontact.com/Global-Telemedicine-and-eHealth-Update--April-2019-.html?soid=1101836993790\&aid=Igjn9NNcZKw (accessed on 9 October 2020).

153. Innovating Pharma with The Blockchain. 2020. Available online: https://isolve.io/ (accessed on 9 October 2020).

154. Brennan, B. iSolve-Innovation for the Drug Supply Chain. 2017. Available online: https://blockchainhealthcarereview.com/ isolve-innovation-drug-supply-chain/ (accessed on 9 October 2020).

155. Democratizing Human Motion Analysis. 2020. Available online: https: / /vay.ai/ (accessed on 9 October 2020).

156. Wauters, R. University of Zurich Spin-off Vay Sports has Launched Its AI-Powered Digital Fitness Coach in Beta. 2019. Available online: https: / tech.eu/brief/university-of-zurich-spin-off-vay-sports-has-launched-its-ai-powered-digital-fitness-coach-inbeta/ (accessed on 9 October 2020). 
157. PokitDok's Platform-as-a-Service Makes it Faster and Easier for Healthcare Organizations to Bring New Applications and Services to Market. 2020. Available online: https:/ / pokitdok.com/ (accessed on 9 October 2020).

158. Change Healthcare Acquires PokitDok Assets. 2018. Available online: https://www.prnewswire.com/news-releases/changehealthcare-acquires-pokitdok-assets-300768002.html (accessed on 9 October 2020).

159. Andriopoulou, F.; Dagiuklas, T.; Orphanoudakis, T. Integrating IoT and fog computing for healthcare service delivery. In Components and Services for IoT Platforms; Springer: Berlin/Heisenberg, Germany, 2017; pp. 213-232.

160. Xu, T.; Wendt, J.B.; Potkonjak, M. Security of IoT systems: Design challenges and opportunities. In Proceedings of the 2014 IEEE/ACM International Conference on Computer-Aided Design (ICCAD), San Jose, CA, USA, 2-6 November 2014; 2014; pp. 417-423.

161. Laplante, P.A.; Laplante, N. The internet of things in healthcare: Potential applications and challenges. It Prof. 2016, 18, 2-4. [CrossRef]

162. Hassanalieragh, M.; Page, A.; Soyata, T.; Sharma, G.; Aktas, M.; Mateos, G.; Kantarci, B.; Andreescu, S. Health monitoring and management using Internet-of-Things (IoT) sensing with cloud-based processing: Opportunities and challenges. In Proceedings of the 2015 IEEE International Conference on Services Computing, New York, NY, USA, 27 June-2 July 2015; pp. $285-292$.

163. Alasmari, S.; Anwar, M. Security \& privacy challenges in IoT-based health cloud. In Proceedings of the 2016 International Conference on Computational Science and Computational Intelligence (CSCI), Las Vegas, NV, USA, 15-17 December 2016; pp. 198-201.

164. Span, P. HIPAA's Use as Code of Silence often Misinterprets the Law. Available online: https://www.nytimes.com/2015/07/21 /health/hipaas-use-as-code-of-silence-often-misinterprets-the-law.html (accessed on 21 September 2020).

165. Mettler, M. Blockchain technology in healthcare: The revolution starts here. In Proceedings of the 2016 IEEE 18th International Conference on e-Health Networking, Applications and Services (Healthcom), Munich, Germany, 14-16 September 2016; pp. 1-3.

166. Prisco, G. The Blockchain for Healthcare: Gem Launches Gem Health Network with Philips Blockchain Lab. Bitcoin Magazine. 2016, p. 26. Available online: https://bitcoinmagazine.com/business/the-blockchain-for-heathcare-gem-launches-gem-healthnetwork-with-philips-blockchain-lab-1461674938 (accessed on 21 September 2020).

167. Williams-Grut, O. Estonia Is Using the Technology behind Bitcoin to Secure 1 Million Health Records. Available online: https: / www.openhealthnews.com/news-clipping/2016-03-03/estonia-using-technology-behind-bitcoin-secure-1-millionhealth-records (accessed on 21 September 2020).

168. Linn, L.A.; Koo, M.B. Blockchain for health data and its potential use in health it and health care related research. In ONC/NIST Use of Blockchain for Healthcare and Research Workshop; ONC/NIST: Gaithersburg, MD, USA, 2016; pp. 1-10.

169. Adopting Blockchain Technology for Electronic Health Record Interoperability. 2020. Available online: https://dokumen.tips/ documents/adopting-blockchain-technology-for-electronic-health-record-.html (accessed on 21 September 2020).

170. Strielkina, A.; Kharchenko, V.; Uzun, D. Availability models for healthcare IoT systems: Classification and research considering attacks on vulnerabilities. In Proceedings of the 2018 IEEE 9th International Conference on Dependable Systems, Services and Technologies (DESSERT), Kyiv, UKraine, 24-27 May 2018; pp. 58-62.

171. Sultan, N. Making use of cloud computing for healthcare provision: Opportunities and challenges. Int. J. Inf. Manag. 2014, 34, 177-184. [CrossRef]

172. Lubamba, C.; Bagula, A. Cyber-healthcare cloud computing interoperability using the HL7-CDA standard. In Proceedings of the 2017 IEEE Symposium on Computers and Communications (ISCC), Crete, Greece, 3-6 July 2017; pp. 105-110.

173. Wu, R.; Ahn, G.J.; Hu, H. Towards HIPAA-compliant healthcare systems. In Proceedings of the 2nd ACM SIGHIT International Health Informatics Symposium, Miami, FL, USA, 28-30 January 2012; pp. 593-602.

174. Seven Compliance of HIPPA. 2018. Available online: https://www.exabeam.com/siem-guide/siem-concepts/hipaa-compliance/ (accessed on 21 September 2020).

175. Shimrat, O. Cloud Computing and Healthcare. San Diego Physician.org, 2009; pp. 26-29. Available online: http: //soundoffcomputing.com/uploads/CloudComputingandHealthcare.pdf (accessed on 21 September 2020). 